\title{
Interplay of disorder and interactions in a flat-band supporting diamond chain
}

\author{
Nilanjan Roy, Ajith Ramachandran, and Auditya Sharma \\ Department of Physics, Indian Institute of Science Education and Research, Bhopal, Madhya Pradesh 462066, India
}

(Received 7 January 2020; revised 18 September 2020; accepted 9 November 2020; published 21 December 2020)

\begin{abstract}
We systematically study the effect of disorder and interactions on a quasi-one-dimensional diamond chain possessing flat bands. Disorder localizes all the single-particle eigenstates, while at low disorder strengths, we obtain weak flat-band based localization (FBL), at high disorder strengths, we see conventional Anderson localization (AL). The compactly localized (CL) eigenstates of flat bands show a persisting oscillatory recurrence in the study of single-particle wave-packet dynamics. For low disorder, a damped oscillatory recurrence behavior is observed which is absent for high disorder. Noninteracting many-particle fermion states also follow the same trend except showing a delocalizing tendency at intermediate disorder due to the fermionic statistics in the system. As interactions are switched on, for the finite sizes that we are able to study, a nonergodic "mixed phase" is observed at low disorder which is separated from the MBL phase at high disorder by a thermal phase at intermediate disorder. A study of many-body nonequilibrium dynamics reinforces these findings.
\end{abstract}

DOI: 10.1103/PhysRevResearch.2.043395

\section{INTRODUCTION}

Translationally invariant Hermitian systems possessing flat bands (FBs) [1-3] exhibit remarkable properties, which emanate from the compactly localized eigenstates (CLS) associated with them. These states are called compact because they reside on a finite volume of the lattice and strictly vanish elsewhere [4-8]. The appearance of flat bands has been theoretically established for lattice models in one [9], two [10], and even three [11] dimensions and realized experimentally with ultracold atoms [12,13], in photonic crystal waveguides [14-16], and exciton-polariton condensates [17]. The physics of such flat-band systems thus hinges on the twin properties of large-scale degeneracy in the dispersion, coupled with extreme localization of associated eigenstates.

Only a very delicate tuning allows for a system to admit exactly flat bands, and the question of how the properties of such systems are modified under different kinds of perturbations is of great interest $[18,19]$. The effect of disorder and interactions is of particular importance because of the pervasive appearance of each of these factors in real systems. A set of studies has been conducted on the effect of disorder in FB systems and the localization-delocalization transitions therein have been understood to some extent $[11,20-25]$. In particular, Ref. [20] points towards the possibility of a different origin for the different phases that emerge as disorder strength is changed. The fate of the FB disordered system when interactions are turned on is of interest too [26-31]. A complexity parametric formulation which could be applicable to a wide range of many-particle interacting flat-band systems

Published by the American Physical Society under the terms of the Creative Commons Attribution 4.0 International license. Further distribution of this work must maintain attribution to the author(s) and the published article's title, journal citation, and DOI. has been considered in Ref. [20]. It is also worth mentioning that localization properties in many-body flat band systems have been recently explored in the context of disorder-free systems [31-34]. In the present paper, we carry out a systematic study of the effect of disorder and interactions on the FB states in a particular system, namely the diamond chain. The investigation of highly degenerate systems under disorder and interactions is challenging, because oftentimes, the degeneracy is only partially lifted, and the study of a single observable is inadequate to explore the impact. Disorder and interactions detune the energy levels as well as modify the characteristics of eigenstates. We therefore conduct a thorough investigation of both the energy spectrum and eigenstates.

We consider a quasi-one-dimensional disordered interacting diamond chain [Fig. 1(a)]. The band structure of the clean, noninteracting diamond chain admits only three flat bands and the system remains essentially in the insulating phase. All the eigenstates in the system are compactly localized on two unit cells and any perturbation is expected to couple these states and increase the volume spanned. We observe three distinct phases of localization in the system: compact localization (CL) at zero disorder, weak flat-band-based localization (FBL) at low disorder, and strong Anderson localization (AL) at high disorder with the localization strength $\mathrm{CL}>\mathrm{AL}>\mathrm{FBL}$. The noninteracting many-fermion system also possesses the FBL and AL phases at low and high disorder strengths, respectively. In the simultaneous presence of disorder and interactions, we find evidence for three distinct phases: a many body localized (MBL) phase at high disorder, a thermal phase at intermediate disorder, and a nonergodic "mixed phase" at low disorder.

To explore the effect of disorder on single-particle states, we consider the level-spacing statistics which is obtained from the inspection of eigenvalues and a variety of quantities: IPR, fidelity, von Neumann entropy, and Shannon entropy, which are obtained from eigenstates. We also explore the dynamics of the system in terms of revival probability and 
(a)

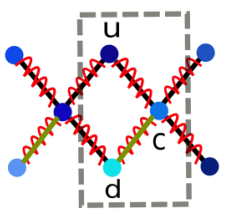

(c)

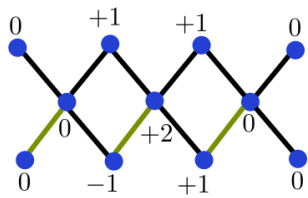

(b)

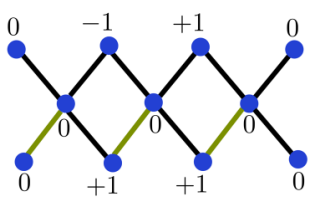

(d)

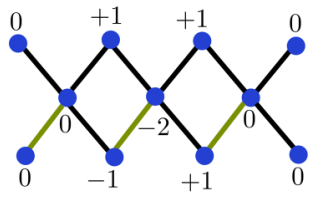

FIG. 1. (a) Schematic of the diamond chain: unit cell contains three sites: up (u), down (d), and central (c) as shown in a box. Nearest neighbor interaction $V$ is represented by red wiggly lines and on-site disorder by the variation in the shades of the color of the dots. [(b)-(d)] The CLS in the noninteracting, disorder-free limit corresponding to $E=0,-2 J$, and $2 J$, respectively, where the numbers assigned to different sites are the probability amplitudes for a single particle.

the imbalance parameter. In the context of noninteracting many fermion states, we study entanglement entropy in the static case and growth dynamics of the entanglement entropy and imbalance parameter. Finally, we study level statistics, many-body IPR and nonequilibrium dynamics of the revival probability, entanglement entropy and imbalance parameter to obtain insights into the properties of many-particle states in the interacting system.

The paper is arranged as follows. Section II describes the details of the model. Section III is devoted to the study of disorder in flat-band systems, which predicts various phases with different localization properties. We then discuss manyparticle states with interactions switched off and explore the phases in the presence of disorder in Sec. IV. In Sec. V, the effect of disorder on the interacting many body system is discussed. The results are summarized in Sec. VI.

\section{THE MODEL}

The Hamiltonian of the quasi-one-dimensional diamond chain can be written as

$$
\hat{H}=\hat{H}_{\text {hop }}+\hat{H}_{\text {os }}+\hat{H}_{\text {int }},
$$

where

$$
\begin{aligned}
\hat{H}_{\mathrm{hop}}= & -J \sum_{i=1}^{N / 3}\left(\hat{u}_{i}^{\dagger} \hat{c}_{i}-\hat{c}_{i}^{\dagger} \hat{d}_{i}+\hat{c}_{i}^{\dagger} \hat{u}_{i+1}+\hat{c}_{i}^{\dagger} \hat{d}_{i+1}\right)+\text { H.c., } \\
\hat{H}_{\mathrm{os}}= & \sum_{i=1}^{N / 3}\left(\zeta_{i}^{u} \hat{u}_{i}^{\dagger} \hat{u}_{i}+\zeta_{i}^{c} \hat{c}_{i}^{\dagger} \hat{c}_{i}+\zeta_{i}^{d} \hat{d}_{i}^{\dagger} \hat{d}_{i}\right), \\
\hat{H}_{\mathrm{int}}= & V \sum_{i=1}^{N / 3}\left(\hat{u}_{i}^{\dagger} \hat{u}_{i} \hat{c}_{i}^{\dagger} \hat{c}_{i}+\hat{c}_{i}^{\dagger} \hat{c}_{i} \hat{d}_{i}^{\dagger} \hat{d}_{i}+\hat{c}_{i}^{\dagger} \hat{c}_{i} \hat{u}_{i+1}^{\dagger} \hat{u}_{i+1}\right. \\
& \left.\hat{+c}_{i}^{\dagger} \hat{c}_{i} \hat{d}_{i+1}^{\dagger} \hat{d}_{i+1}\right)+ \text { H.c. }
\end{aligned}
$$

Here, $\hat{u}_{i}^{\dagger}, \hat{c}_{i}^{\dagger}$, and $\hat{d}_{i}^{\dagger}$ are the fermionic creation operators acting at the $u$ (up), c (center), and d (down) sites respectively in the $i$ th unit cell, as schematically shown in Fig. 1(a). The total number of sites is denoted by $N$ and is taken as a multiple of 3 , because of the unit cell structure. The hopping amplitude $J$ and interaction $V$ are nonzero for nearest neighbors [represented as sites connected by lines in Fig. 1(a)] and zero otherwise. $\zeta_{i}^{\alpha}(\alpha=u, c, d)$ denotes the strength of the on-site disorder chosen from a uniform random distribution $[-\Delta, \Delta]$.

\section{A. Compact localization}

In the absence of disorder and interaction $\left(\zeta_{i}^{\alpha}=0, V=0\right)$, the band structure of the diamond chain contains three flat bands at $E=0, \pm 2 J$. Lattices with all bands flat are rare and the diamond chain is carefully tuned to admit this intriguing feature [27]. Conventional wisdom implies that the eigenstates of a translationally invariant system are Bloch states which span the entire lattice and which carry the delocalization characteristics associated with the dispersion of the energy bands. The dispersionless property of the flat bands on the one hand implies zero group velocity for the states and on the other hand provides a convenient representation where the eigenstates are compactly localized. Due to the large scale degeneracy, the basis can be chosen in multiple ways, however one can always look for a basis such that the eigenstates occupy the smallest volume in the lattice. Once one such state has been identified, the translational invariance of the Hamiltonian can be utilized to obtain others states belonging to the flat bands. Although suitable linear combinations of the compact localized eigenstates can of course recover the Bloch state representation, it is the properties of the CLS that make these systems most interesting.

The states belonging to all three flat bands in the diamond chain can be represented as different compact localized states. Figures 1(b)-1(d) shows one CLS belonging to each band where \pm 1 corresponds to the amplitudes (not normalized) of the states on the sites. The origin of compactness of these states is the destructive interference at the neighboring sites which effectively confines the states within a finite volume. For the diamond chain Hamiltonian in Eq. (1) with no disorder and interaction, the smallest possible volume for a CLS is two unit cells as shown in Figs. 1(b)-1(d). The other states corresponding to each flat band can be obtained by shifting these states along the lattice utilizing the translational invariance of the Hamiltonian. It can be shown that the states identified in this particular case lack orthogonality although they form a complete set. The localization properties of the CLS are special as they are observed in a translationally invariant system and span very small volume of the lattice with an abrupt boundary without any tail in spread. This is to be contrasted with Anderson localization (AL) observed in disordered systems where the states exhibit an exponential tail. Hence the CLS possess stronger localization compared to that seen in AL.

\section{EFFECT OF DISORDER ON SINGLE-PARTICLE PROPERTIES}

In this section, we analyze the eigenvalues and singleparticle eigenfunctions to study the effect of disorder. The level-spacing ratio and level-spacing distribution are obtained 

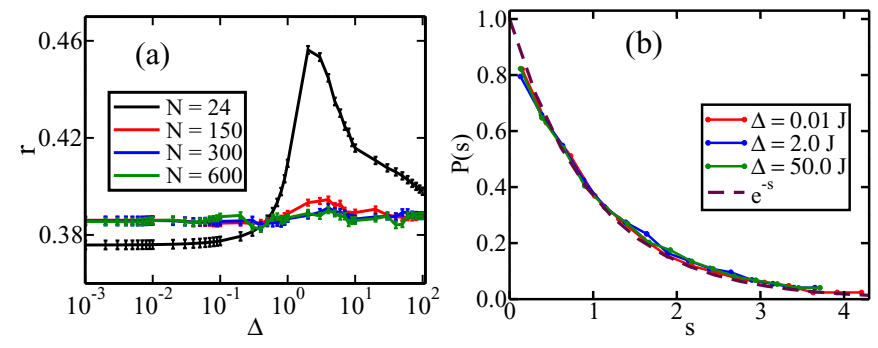

FIG. 2. (a) Level-spacing ratio $r$ vs disorder strength $\Delta$ (in units of $J$ ) for increasing values of system size $N$. (b) Level-spacing distribution for increasing disorder strength for $N=300$. The dimensionless level-spacings $s$ are obtained by dividing the original level-spacings by the mean level-spacing of the spectrum. The number of disorder realizations varies for different system sizes, but all of them have at least 100 samples of disorder.

from the single-particle eigenvalues, while the inverse participation ratio (IPR), fidelity, von Neumann entropy, and Shannon entropy are calculated from the eigenfunctions. Nonequilibrium dynamics throws further light on some of these single-particle properties.

\section{A. Level-spacing statistics}

The level-spacing ratio $r$ is calculated from the eigenvalues of the disordered Hamiltonian and is defined as

$$
r=\frac{1}{N-1} \sum_{k=1}^{N-1} \frac{\min \left[s_{k}, s_{k+1}\right]}{\max \left[s_{k}, s_{k+1}\right]},
$$

where energy level spacing $s_{k}=E_{k+1}-E_{k}$ with singleparticle energies $E_{k}$ 's organized in the ascending order for a system size of $N$ sites and with an implicit average over realizations of random disorder assumed. In the delocalized and localized phases $r$ is expected to be approximately 0.528 and 0.386 , respectively. Figure 2(a) shows the dependence of $r$ on the strength of disorder, for various system sizes. For a small system size $N=24, r$ is close to 0.386 at very small disorders $\Delta(\Delta \ll J)$ and seems to show a tendency to deviate from 0.386 for disorder strength comparable to hopping strength $J$. However, this is seen to be a finite-size effect and for larger system sizes, the level-spacing ratio $r$ remains at 0.386 in the full range of the disorder strength $\Delta$. This indicates that the system is localized in the presence of disorder. In the absence of disorder, the level-spacing ratio becomes unintelligible due to the massive degeneracy.

We also study the energy level-spacing distribution function $P(s)$, where $s_{i}$ 's are made dimensionless by dividing the original level spacings by the mean level-spacing of the spectrum such that $\int P(s) d s=1$. For a real Hamiltonian, in the delocalized phase, the spectral statistics is $G O E$, i.e., $P(s)=\frac{\pi}{2} s e^{-\frac{\pi}{4} s^{2}}$, which yields $r \approx 0.528$. In the localized phase, the spectral statistics is Poissonian $P(s)=e^{-s}$ yielding $r \approx 0.386$. In Fig. 2(b), we observe that the energy levelspacing distribution follows Poissonian spectral statistics for all disorder strengths confirming that the system is localized irrespective of the strength of disorder. The introduction of disorder into the insulating all-band flat diamond chain keeps the system in the insulating phase itself. We further explore

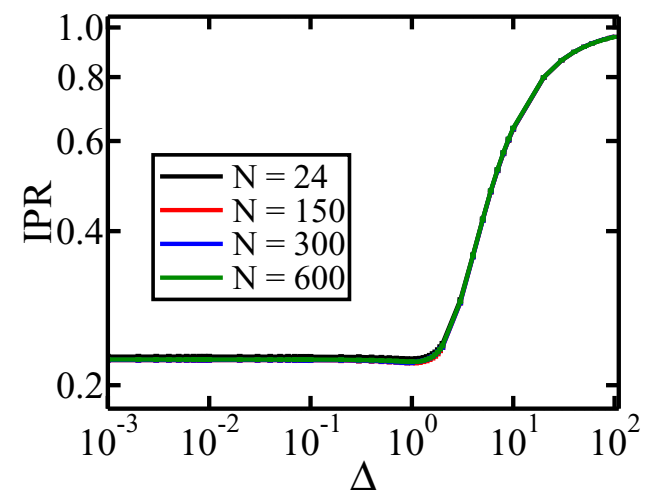

FIG. 3. The average IPR of all the single-particle eigenstates vs disorder for increasing values of system size $N$. The number of disorder realizations varies for different system sizes, but all of them have at least 100 samples of disorder.

the localization properties of the system at different disorder strengths in detail through a study of the eigenstates.

\section{B. Inverse participation ratio}

The inverse participation ratio (IPR) is defined as

$$
I_{k}=\sum_{i=1}^{\frac{N}{3}} \sum_{\alpha=u, c, d}\left|\psi_{k}(i, \alpha)\right|^{4},
$$

where the $k$ th normalized single-particle eigenstate $\left|\psi_{k}\right\rangle=$ $\sum_{i, \alpha} \psi_{k}(i, \alpha)|i, \alpha\rangle$ is written in terms of the Wannier basis $|i, \alpha\rangle$, representing the state of a single particle localized at the site $\alpha(\alpha=u, c, d)$ in the $i$ th unit cell of the lattice. For a perfectly delocalized eigenstate, $I_{k}$ scales as $1 / N$, while for a single-site localized eigenstate, $I_{k}=1$ and at criticality $I_{k}$ shows intermediate behavior.

The IPR averaged over all the eigenstates plotted in Fig. 3 reveals two distinct localization phases: a weak flat-band based localization (FBL) at low disorder with low IPR and a strong Anderson localization (AL) at high disorder with an IPR close to one. We observe a cross-over from the FBL to AL at $\Delta=2 J$. In the low-disorder region, the states within one band hybridize leading to the FBL and as disorder strength increases states from all bands hybridize leading to the conventional AL. Thus, even though the system is localized, there could be substantial difference in the localization properties at different disorder strengths. How different are the states at low and high disorder regions is an interesting question and we explore this through the study of fidelity in the next section.

\section{Fidelity}

To understand the nature of the single-particle eigenstates in the presence of disorder, the average fidelity or overlap between the eigenstates corresponding to $\Delta=0.001 \mathrm{~J}$ and those corresponding to higher values of $\Delta>0.001 \mathrm{~J}$ is calculated. Fidelity between the $k$ th eigenstates corresponding to two values of $\Delta$ is defined as

$$
F_{12}^{k}=\left|\left\langle\psi_{k}\left(\Delta_{1}\right) \mid \psi_{k}\left(\Delta_{2}\right)\right\rangle\right|^{2} .
$$




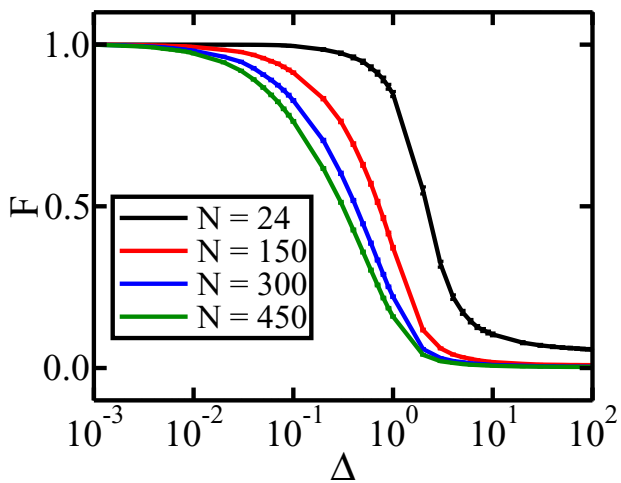

FIG. 4. Spectrum averaged fidelity $F$ as a function of the disorder strength $\Delta$ (in units of $J$ ) for increasing system sizes $N$. The number of disorder realizations varies for different system sizes, but all of them have at least 100 samples of disorder.

The two different phases of localization FBL and AL are evident from the spectrum averaged fidelity $F=\left\langle F^{k}\right\rangle$ shown in Fig. 4. The characteristics of eigenstates in the FBL phase and the AL phase are different from one another. Fidelity provides a comparison between each eigenstate at different disorder strengths. One main observation we make through this study is that, even though the IPR in the weak FBL states are obtained to be independent of disorder [Fig. 3(a)], a closer examination of fidelity (Fig. 4) reveals that the nature of eigenstates in fact changes substantially with disorder strength. This is in contrast to conventional AL where the eigenstates are frozen such that the variation of $F$ with respect to disorder strength is minimal. Moreover, in the FBL phase, the fidelity seems to have a strong dependence on system size $N$, in sharp contrast to the AL phase.

\section{Von Neumann entropy and Shannon entropy}

The von Neumann entropy is a good measure to explore localization phenomena in quantum systems [35]. In this work, we aim to calculate von Neumann entropy connected to a single site. A single particle has two local states $|0\rangle_{i, \alpha}$ and $|1\rangle_{i, \alpha}$ at the site $\alpha$ of the $i$ th unit cell and hence the local density matrix $\rho_{k}^{i \alpha}$ for the $k$ th eigenstate can be written as [36]

$$
\rho_{k}^{i \alpha}=\left|\psi_{k}(i, \alpha)\right|^{2}|1\rangle_{i \alpha}\left\langle\left. 1\right|_{i \alpha}+\left(1-\left|\psi_{k}(i, \alpha)\right|^{2}\right) \mid 0\right\rangle_{i \alpha}\left\langle\left. 0\right|_{i \alpha} .\right.
$$

The von Neumann entropy associated with site $i$ is given by

$$
\begin{aligned}
S_{k}^{i \alpha}= & -\left|\psi_{k}(i, \alpha)\right|^{2} \log _{2}\left(\left|\psi_{k}(i, \alpha)\right|^{2}\right) \\
& -\left(1-\left|\psi_{k}(i, \alpha)\right|^{2}\right) \log _{2}\left(1-\left|\psi_{k}(i, \alpha)\right|^{2}\right) .
\end{aligned}
$$

In a delocalized eigenstate $\left|\psi_{k}(i, \alpha)\right|^{2}=1 / N$ and hence $S_{k}^{i \alpha} \approx \frac{1}{N} \log _{2} N+\frac{1}{N}$ for large values of $N$ whereas for a single-site localization $S_{k}^{i \alpha}=0$. The contributions from all sites for a particular eigenstate are given by $S_{k}=\sum_{i, \alpha} S_{k}^{i \alpha}$. Thus the average von Neumann entropy over all the eigenstates is defined as

$$
S^{\mathrm{sp}}=\frac{\sum_{k=1}^{N} S_{k}}{N} .
$$

For large values of $N, S^{\mathrm{sp}} \approx\left(\log _{2} N+1\right)$ in the delocalized phase whereas $S^{\mathrm{sp}} \approx 0$ in an extremely (single-site) localized
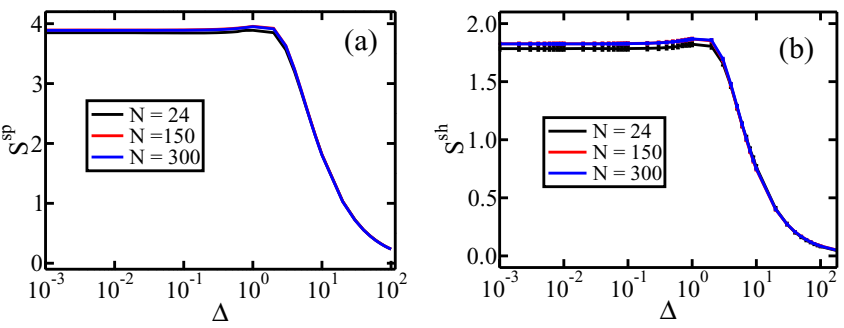

FIG. 5. (a) The von Neumann entropy averaged over all the eigenstates vs disorder for increasing system sizes $N$. (b) Shannon entropy $S^{\text {sh }}$, averaged over all the eigenstates as a function of disorder strength $\Delta$ (in units of $J$ ) for increasing $N$. The number of disorder realizations varies for different system sizes, but all of them have at least 100 samples of disorder.

phase. For the critical phases, $S^{\mathrm{sp}}$ can take any intermediate values. From Fig. 5, which shows the von Neumann entropy $S^{\mathrm{sp}}$ as a function of disorder $\Delta$, it can be seen that $S^{\mathrm{sp}}$ is less than $\left(\log _{2} N+1\right)$ for all disorder strengths and system sizes, pointing towards localization. However, the higher values of von Neumann entropy at low disorder strengths compared to those at high disorder strengths indicate the weakly localized FBL phase for small $\Delta$ as compared to the strong AL phase for large $\Delta$. Also we note that similar to IPR, the value of spectrum averaged $S^{\mathrm{sp}}$ remains disorder strength independent in the FBL phase.

For the $k$ th eigenstate, the Shannon entropy is given by

$$
S_{k}^{\mathrm{sh}}=-\sum_{i, \alpha}\left|\psi_{k}(i, \alpha)\right|^{2} \ln \left|\psi_{k}(i, \alpha)\right|^{2} .
$$

The Shannon entropy averaged over all the eigenstates $S^{\text {sh }}=$ $\left\langle S_{k}^{\text {sh }}\right\rangle$ is shown in Fig. 5(b). For a perfectly delocalized state, $S^{\text {sh }}=\ln N$, whereas for a perfectly localized state, $S^{\text {sh }}$ is 0 , where the system size independence implies localization. The figure shows the presence of two localized phases: FBL phase with higher $S^{\text {sh }}$ and AL phase with lower $S^{\text {sh }}$, consistent with the observation from IPR.

\section{E. Nonequilibrium dynamics of a single particle}

Next we study nonequilibrium properties of the system by keeping a single particle initially at some lattice site $m_{0}$, i.e.,
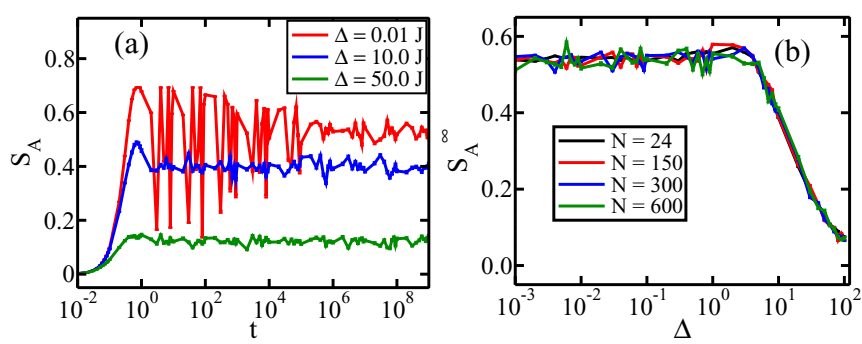

FIG. 6. (a) The entanglement entropy $S_{A}$ as a function of time (in units of $J^{-1}$ ) for a single particle for system size $N=600$ and different disorder strengths. (b) The saturation value $S_{A}^{\infty}$ as a function of $\Delta$ (in units of $J$ ) for increasing values of $N$. The number of disorder realizations varies for different plots, but all of them have at least 100 samples of disorder. 

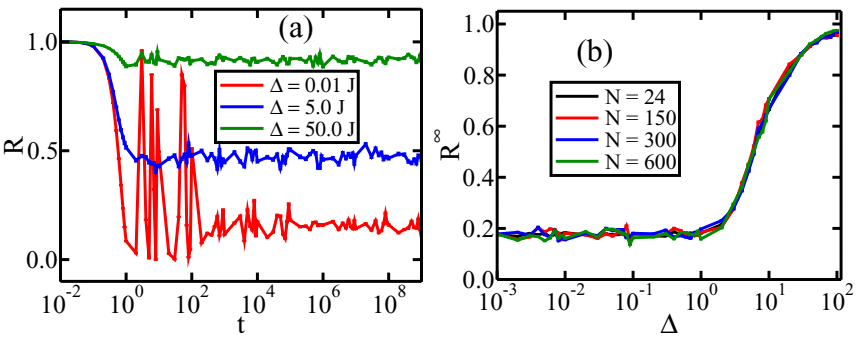

FIG. 7. (a) The revival probability $R$ as a function of time (in units of $J^{-1}$ ) for a single particle for system size $N=600$ and increasing disorder strength $\Delta$. (b) The saturation value $R^{\infty}$ as a function of $\Delta$ (in units of $J$ ) for increasing values of $N$. The number of disorder realizations varies for different plots, but all of them have at least 100 samples of disorder.

$\left|\psi_{\text {in }}\right\rangle=\left|m_{0}\right\rangle$. Here we arbitrarily choose $m_{0}$ to be a $d$ site, although the other choices should yield similar results. We calculate various dynamical quantities such as the entanglement entropy, revival probability and width of the single-particle wave packet in the following.

The entanglement entropy of a single particle is given by [36,37] $S_{A}(t)=-p_{A}(t) \ln p_{A}(t)+\left(1-p_{A}(t)\right) \ln \left(1-p_{A}(t)\right)$, where $p_{A}(t)=\sum_{i, \alpha \in A}|\psi(i, \alpha)|^{2}$, is the instantaneous probability of finding the particle inside subsystem $A$ which is taken to be half of the full system. The dynamics of entanglement entropy is shown in Fig. 6(a) for different disorder strengths $\Delta$. For very low disorder $(\Delta=0.01 J)$, after a superballistic transient, a damped oscillatory behavior is observed for the entanglement entropy (see Appendix A for the zero-disorder case) followed by saturation. This oscillatory behavior vanishes at high disorder strength (e.g., $\Delta \geqslant 10 J$ ). The saturation value $S_{A}^{\infty}$ is plotted as a function of $\Delta$ for increasing system sizes in Fig. 6(b), which clearly reflects the presence of two localized regimes; weak flat-band based localization and strong Anderson localization.

We also study revival probability $R(t)=\left|\left\langle\psi_{\text {in }} \mid \psi_{t}\right\rangle\right|^{2}$ of finding the particle at the initial site. The dynamics of the revival probability $R$ shown in Fig. 7(a) indicates damped oscillatory behavior in the tiny disorder regime $\Delta=0.01 \mathrm{~J}$ whereas this characteristic vanishes in the high disorder regime $\Delta>5.0 \mathrm{~J}$. The saturation value $R^{\infty}$ of revival proba-
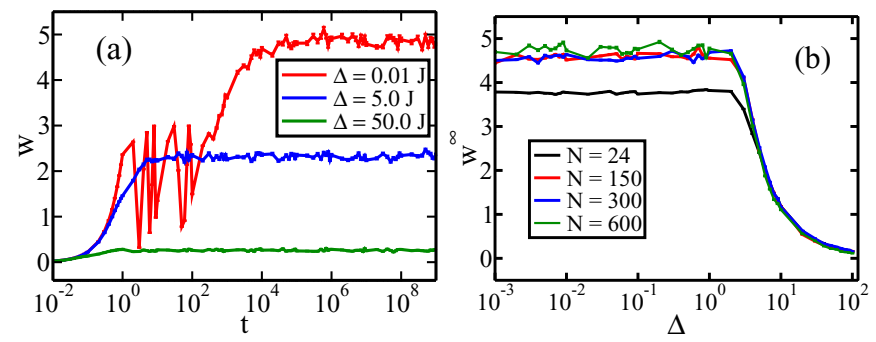

FIG. 8. (a) The dynamics of width of the wave packet $w$ as a function of time (in units of $J^{-1}$ ) for a single particle for system size $N=600$ and different disorder strengths. (b) The saturation value $w^{\infty}$ as a function of $\Delta$ (in units of $J$ ) for increasing values of $N$. The number of disorder realizations varies for different plots, but all of them have at least 100 samples of disorder.

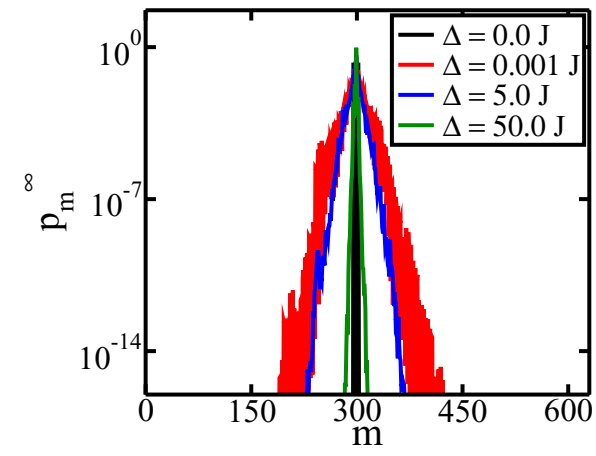

FIG. 9. The on-site occupation probability of a single particle in the long-time limit, denoted as $p_{m}^{\infty}$ (in a logarithmic scale) at site $m$ for $N=600$ and disorder strength $\Delta=0,0.001 \mathrm{~J}, 5.0 \mathrm{~J}$, and $50.0 \mathrm{~J}$, respectively. In this plot, number of disorder realizations is 200 .

bility [Fig. 7(b)] turns out to be independent of system size $N$ for all disorder strengths, which is characteristic of a localized phase. The figure clearly shows the presence of two localized phases thereby supporting the results obtained in the static calculations.

The width of the wave packet is defined as $w=$ $\sqrt{\left\langle\hat{x}^{2}\right\rangle-\langle\hat{x}\rangle^{2}}$, where $\hat{x}$ is the position operator. The dynamics of $w$ is shown in Fig. 8(a). After a ballistic transient, there is a damped oscillation due to recurrence for $\Delta=0.01 \mathrm{~J}$, followed by an extended sub-diffusive regime before it reaches saturation. For large values of disorder $\Delta>5.0 J$, after the ballistic transient there is a short sub-diffusive regime followed by saturation. The oscillatory recurrence regime is absent in this case. The saturation value $w^{\infty}$ as a function of $\Delta$ shown in Fig. 8(b) clearly indicates weak flat-band based localization for small disorder and strong Anderson localization for large disorder respectively. We note that $w^{\infty}$ becomes independent of $N$ for large values of $N$, for all values of $\Delta$, which is a signature of localization.

In Fig. 9, we show the on-site occupancy of a single particle in the long-time limit. For $\Delta=0$, the wave function is extremely localized within very few sites reflecting the localization properties of CLS. Turning on a tiny disorder $\Delta=0.001 J$ makes the occupation probability function $p_{m}^{\infty}$ extended in space with exponential tails. Increasing the disorder further to $\Delta>5.0 \mathrm{~J}$ shrinks the function with sharper exponential tails. This establishes the hierarchy for localized phases: $\mathrm{CL}>\mathrm{AL}>\mathrm{FBL}$ in terms of the strength of localization.

\section{NONINTERACTING SPINLESS FERMIONS}

In this section, we report the effect of disorder on noninteracting spinless fermions in the diamond chain. This should be viewed as a bridge between the single-particle physics of the previous section, and interacting many-particle physics of the following section. In this entire section, we set the interaction strength to zero $(V=0)$, and the filling fraction to $\frac{1}{6}$. Using correlation matrix techniques, we compute entanglement entropy and imbalance parameter, which help in revealing the characteristics of the system under disorder. 

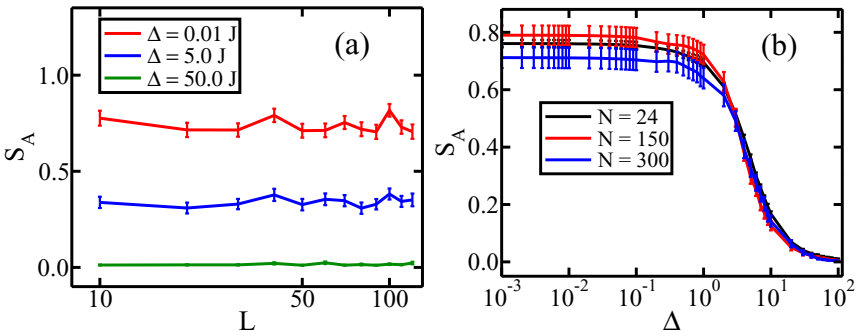

FIG. 10. (a) The subsystem size $L$ dependence of the entanglement entropy $S_{A}$ of the fermionic ground state for increasing values of disorder strength $\Delta$ for system size $N=300$. (b) The $S_{A}$ as a function of $\Delta$ (in units of $J$ ) for increasing values of $N$ with $L=N / 2$. For all the plots, the number of disorder realizations is 100 and filling fraction of the noninteracting fermions is $1 / 6$.

\section{A. Fermionic entanglement entropy of the ground state}

Entanglement entropy is a useful quantity to distinguish between different many-body quantum phases in the context of localization [38,39]. The entanglement entropy between two subsystems is given by $S_{A}=-\operatorname{Tr}\left(\rho_{A} \ln \rho_{A}\right)$, where the reduced density matrix $\rho_{A}=\operatorname{Tr}_{B}(\rho)$ is obtained from the many body ground state $\left|\Psi_{0}\right\rangle$ through the density matrix $\rho=\left|\Psi_{0}\right\rangle\left\langle\Psi_{0}\right|$. The computation of entanglement entropy for many-body ground states of noninteracting spinless fermions is greatly simplified with the help of free fermionic correlation matrix techniques [40,41]. For a single Slater determinant ground state, using Wick's theorem, the reduced density matrix can be represented as $\rho_{A}=\frac{e^{-H_{A}}}{Z}$, where $H_{A}$ is the entanglement Hamiltonian (that is guaranteed to have a quadratic form), and $Z$ is obtained from the condition $\operatorname{Tr}\left(\rho_{A}\right)=1$. The information contained in the reduced density matrix of size $2^{L} \times 2^{L}$ can be captured in terms of the correlation matrix $C$ of size $L \times L[40]$ within the subsystem A, where $C_{i j}^{\alpha \beta}=\left\langle\alpha_{i}^{\dagger} \beta_{j}\right\rangle$ with $\alpha, \beta \in\{u, c, d\}$, and $i, j$ denoting the unit cell index. The correlation matrix and the entanglement Hamiltonian are related by [40-42] $C=\frac{1}{e^{H_{A}}+1}$. Using this relation, the entanglement entropy for free fermions is given by $[41,42]$

$$
S_{A}=-\sum_{m=1}^{L}\left[\zeta_{m} \ln \zeta_{m}+\left(1-\zeta_{m}\right) \ln \left(1-\zeta_{m}\right)\right],
$$

where $\zeta_{m}$ 's are the eigenvalues of the correlation matrix $C$.

Subsystem scaling of entanglement entropy has been used to distinguish quantum phases $[38,39,43,44]$. For free fermions in $d$ dimensions, typically $S_{A} \propto L^{d-1} \ln L$ [45] in metallic phases whereas $S_{A} \propto L^{d-1}$ (also known as area-law) in localized phases in the presence of disorder. Since localized systems do not care about the boundary of two subsystems, generally the "area law" can be used as the defining signature to detect such phases. For the present system, we show the subsystem size dependence of $S_{A}$ of noninteracting fermions at $1 / 6$ filling in Fig. 10(a). Here subsystem size $L$ is the number of sites belonging to subsystem A. All the plots abide by the area-law for all values of $\Delta$, indicating localization. As $\Delta$ is increased $S_{A}$ shows [Fig. 10(b)], a crossover from a high value to a low value independent of system size pointing towards the presence of two localized phases in the many-particle ground state. We observe that the low- $\Delta$ region
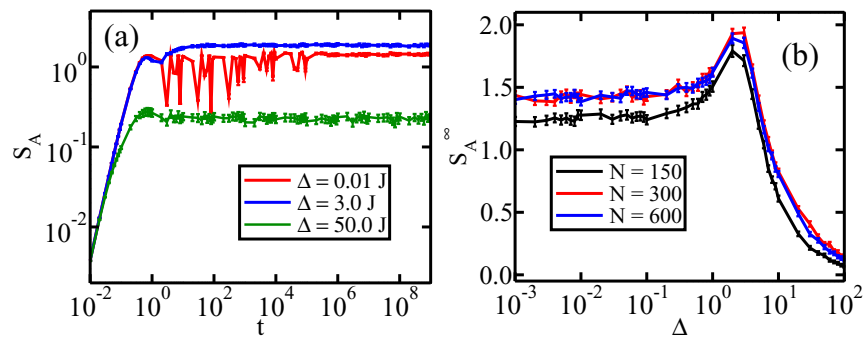

FIG. 11. (a) The growth of the entanglement entropy $S_{A}$ with time (in units of $J^{-1}$ ) for system size $N=600$ and disorder strength $\Delta=0.01 \mathrm{~J}, 3.0 \mathrm{~J}$, and $50.0 \mathrm{~J}$, respectively. (b) The saturation value $S_{A}^{\infty}$ as a function of $\Delta$ (in units of $J$ ) for increasing values of $N$. The number of realizations of disorder is 200 .

corresponding to FBL has a small (compared to thermal value) finite value of entanglement, which is independent of system size, and hence characteristic of a localized phase. However at large $\Delta$, the entanglement entropy is almost zero, indicating the AL phase.

\section{B. Nonequilibrium dynamics of noninteracting fermions}

Next, we study the nonequilibrium transport properties of noninteracting fermions in the system. We consider an initial many-body state of the following form: $\left|\Psi_{i n}\right\rangle=\prod_{i=1}^{N / 3} \hat{c}_{2 i}^{\dagger}|0\rangle$, which is a product state of fermions in which $c$ site of alternating unit cells is occupied by a single particle leading to a filling fraction of $1 / 6$. For this type of a choice of the initial state, Wick's theorem works and hence the free fermionic techniques [40-42] can be applied to study the growth dynamics of the entanglement entropy. After a superballistic transient [Fig. 11(a)] an oscillatory regime (see Appendix A) is observed for $\Delta=0.01 \mathrm{~J}$, which is absent in case of $\Delta=3.0 \mathrm{~J}$ and $50.0 \mathrm{~J}$. It is notable that the long-time saturation value of $S_{A}$ is higher for $\Delta \approx 3.0 \mathrm{~J}$ as compared to those for $\Delta=0.01 \mathrm{~J}$ and $50.0 \mathrm{~J}$. The hump in the saturation value $S_{A}^{\infty}$ [Fig. 11(b)] in the intermediate range of values of $\Delta$ is due to fermionic statistics, and is absent in the single-particle case.

We have also studied the imbalance parameter, a more experimentally relevant quantity, which is given by

$$
I_{b}(t)=\frac{\sum_{i=1}^{N / 3}(-1)^{i} n_{i}^{c}-n_{i}^{u}-n_{i}^{d}}{\sum_{i=1}^{N / 3} n_{i}^{c}+n_{i}^{u}+n_{i}^{d}},
$$

where $n_{i}^{\alpha}$ is the occupancy of fermions at the site $\alpha$ of the $i$ th unit cell where $\alpha \in u, c, d$. Essentially the numerator is the difference in occupancies between the initially occupied sites and the initially unoccupied sites whereas the denominator simply sums up to the total number of particles $N_{p}$. For a perfectly delocalized many-body state $I_{b}=-2 / 3$, whereas for a perfectly localized many-body state $I_{b}=1$.

The dynamics of the imbalance parameter $I_{b}(t)$ [Fig. 12(a)] shows a damped oscillatory regime for $\Delta=0.01 \mathrm{~J}$ and this behavior is absent for $\Delta=3.0 \mathrm{~J}$ and $50.0 \mathrm{~J}$ pointing out the characteristic difference between the small and large $\Delta$ regimes. The saturation value of $I_{b}$ for $\Delta=3.0 \mathrm{~J}$ is low compared to those for $\Delta=0.01 \mathrm{~J}$ and $50.0 \mathrm{~J}$ indicating the possibility of an intermediate phase. To explore this further, the saturation value $I_{b}^{\infty}$ as a function of disorder strength $\Delta$ 

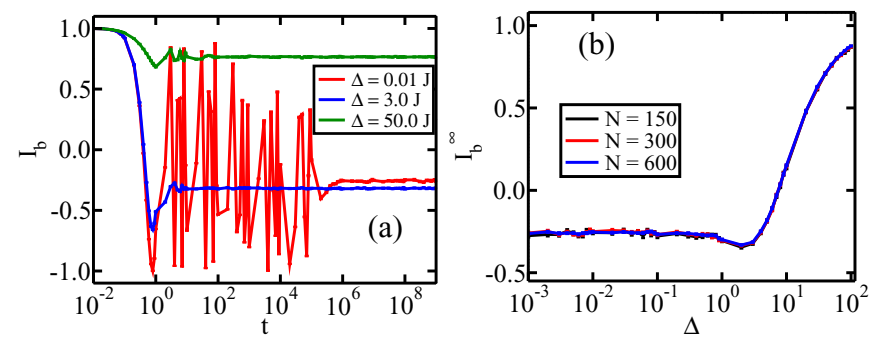

FIG. 12. (a) The imbalance parameter $I_{b}$ as a funtion of time (in units of $J^{-1}$ ) for system size $N=600$ and disorder strength $\Delta=$ $0.01 \mathrm{~J}, 3.0 \mathrm{~J}$, and $50.0 \mathrm{~J}$ respectively. (b) The saturation value $I_{b}^{\infty}$ as a function of $\Delta$ (in units of $J$ ) for increasing values of $N$. The number of realizations of disorder is 200 .

for different system sizes $N$ is shown in Fig. 12(b). A dip is observed in the intermediate range of disorder strength $\Delta$, where a hump is observed for the entanglement entropy. Such dips may be signatures of delocalization but on the other hand, system size $N$ independence of $I_{b}^{\infty}$ at dips is a signature of localization. In order to make a conclusive comment on delocalization/localization one needs to look at the energy level-statistics, which is rather difficult to perform for large system sizes $N$ using the exact diagonalization method. Such dips are absent in single-particle properties, but seem to show up in the noninteracting many-particle properties, and will also be encountered in the interacting many-particle properties in the next section. Therefore, as we might expect, the noninteracting many-particle properties show features in between those of single-particle and interacting many-particle states.

\section{INTERACTING SPINLESS FERMIONS}

In this section, we develop an understanding of the transport properties of interacting spinless fermions in the disordered diamond chain. We employ the level-spacing ratio and spectrum averaged many-particle inverse participation ratio to capture the interaction induced $(V \neq 0)$ crossovers in this disordered model.

\section{A. Level-spacing statistics}

Level-spacing ratio defined in Eq. (3) is studied using the energy spectra $\left(\mathcal{E}_{1}, \mathcal{E}_{2}, \mathcal{E}_{3}, \ldots, \mathcal{E}_{D}\right)$ of the interacting
Hamiltonian via exact diagonalization, where $D=\left(\begin{array}{l}N \\ N_{p}\end{array}\right)$ is the dimension of the particle-number constrained Hilbert space and $v=N_{p} / N$ with $N_{p}$ and $N$ being the number of fermions and the system size, respectively. The level-spacing ratio $r$ as a function of disorder strength $\Delta$ for different $V$ is shown in Figs. 13(a), 13(b) and 13(c) for $N=18$ and filling fraction of fermions $v=1 / 9,1 / 6$, and $2 / 9$, respectively. We observe that the three phases become most distinct for the highest filling as shown in Fig. 13(c): an MBL phase at high disorder strengths $\Delta>10.0 J$ with $r=0.386$, a thermal phase for intermediate disorder strengths $\Delta=\mathcal{O}(J)$ and for $V=\mathcal{O}(J)$ with $r$ approaching 0.528 , and a "mixed phase" at low disorder strengths $\Delta \ll J$ with $r$ neither 0.528 nor 0.386 . In the low disorder region, when the interaction $V \approx \Delta$, the amount of delocalization increases in the system in the mixed phase, as $r$ shows a peak (see Sec. VC). For the intermediate disorder strengths, the system delocalizes with increasing filling fraction and the peak of $r$ [Figs. 13(b) and 13(c)] almost approaches 0.528 at $V \approx \Delta$ as the number of fermions is increased. We also observe that the peak corresponding to delocalization moves towards a lower value of disorder strength $\Delta$ as the number of fermions increases. Thus, the filling fraction appears to aid the repulsive effects of Pauli exclusion. What happens in the low $\Delta$ regime as a function of filling fraction will be addressed in detail later.

We also show the system size dependence of level-spacing ratio $r$ versus $\Delta$ for $V=1.0 J$ and $v=1 / 6$ in Fig. 14. Here, as the system size $N$ increases, the particle number $N_{p}$ also increases to maintain $v=1 / 6$. As $N$ increases, we observe that the phase at high disorder strengths gets more sharply defined. A substantial delocalization is observed in the intermediate disorder region and $r$ seems to approach 0.528 with increase in system size. In the low $\Delta$ regime, values of $r$ are much lower than 0.386 for $N=12$. This happens typically due to restoration of the crystal momentum conservation [46], which has been recently addressed in the literature for two interacting particles [47]. As system size increases $\left(N_{p}\right.$ increases accordingly to fix $\left.v\right), r$ increases and becomes 0.386 for $N=24$, although it is not very clear whether $r$ will increase even further with $N$. We infer that it is a mixed phase with the localized eigenstates being dominant (see Fig. 18 and the discussion in the Sec. V C). The three phases become more well established with increasing $v$ as can be seen from Fig. 13.
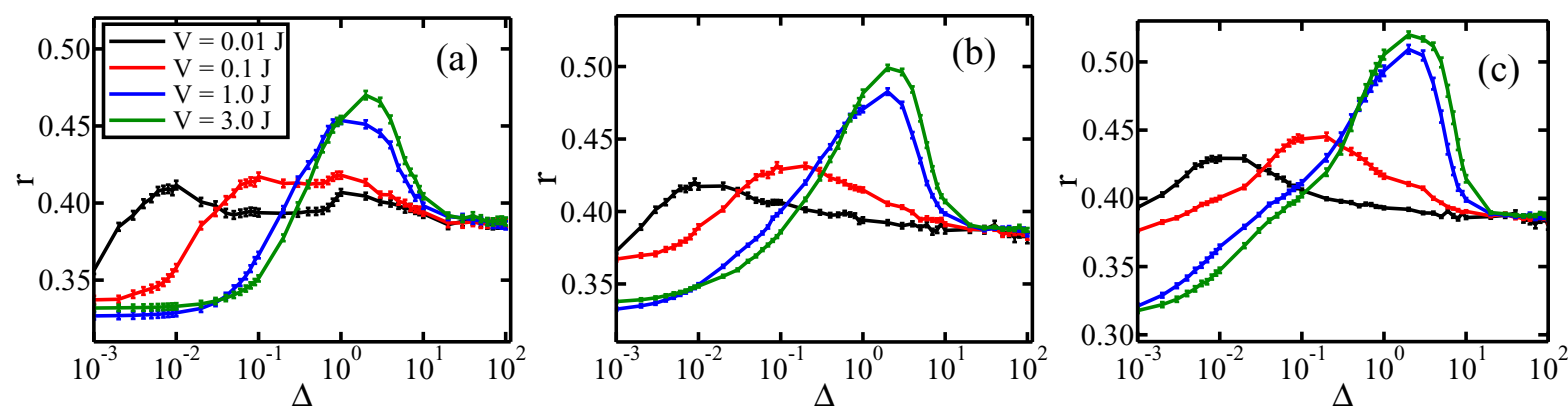

FIG. 13. [(a)-(c)] The level-spacing ratio $r$ as a function of disorder strength $\Delta$ (in units of $J$ ) for increasing interaction strength $V$ for fermionic filling fraction (a) $1 / 9$, (b) $1 / 6$, and (c) $2 / 9$, respectively. For all the plots system size $N=18$. Number of disorder realizations are 500,200 , and 100 for $1 / 9,1 / 6$, and $2 / 9$ fillings, respectively. 


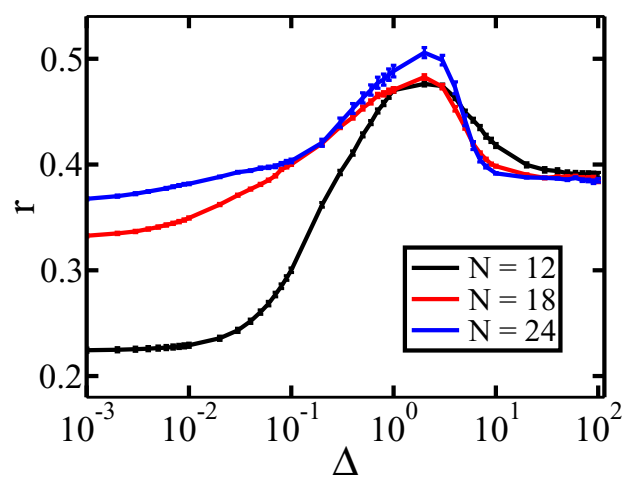

FIG. 14. The level-spacing ratio $r$ as a function of disorder strength $\Delta$ (in units of $J$ ) for fixed $V=1.0 J$ and system sizes $N=12,18$, and 24 for filling fraction of fermions $v=1 / 6$. The quantities are averaged over 500,200, and 30 disorder realizations for $N=12,18$, and 24, respectively for fermion filling $v=1 / 6$.

\section{B. Many-particle inverse participation ratio}

Expanding the normalized eigenstate $|\Psi\rangle$ in the particlenumber constrained space as $|\Psi\rangle=\sum_{n=1}^{D} C_{n}|n\rangle$, the manyparticle inverse participation ratio (MIPR) is defined as

$$
\operatorname{MIPR}=\sum_{n=1}^{D}\left|C_{n}\right|^{4} .
$$

For an extremely localized eigenstate MIPR $=1$ whereas for a perfect delocalized eigenstate MIPR $=1 / D$. The spectrumaveraged MIPR as a function of $\Delta$ for different interaction strengths $V$ is shown in Figs. 15(a), 15(b) and 15(c) for system size $N=18$ and filling $v=1 / 9,1 / 6$, and 2/9, respectively. The plots also confirm the three distinct phases: MBL, thermal, and mixed observed during the examination of levelspacing ratio $r$. The MBL phase possesses high MIPR $\approx 1$, while the thermal phase has a low MIPR representing a dip at $\Delta \approx 2 J$. The mixed phase is observed at the low disorder strengths $\Delta \ll J$. In the mixed phase, another small dip is observed at $V \approx \Delta$ corresponding to an increment in the amount of delocalization.

Apparently the dips of MIPR at the intermediate disorder strength become deeper with increasing filling fraction indicating more delocalization. To confirm this we carry out a careful scaling analysis with $D$.
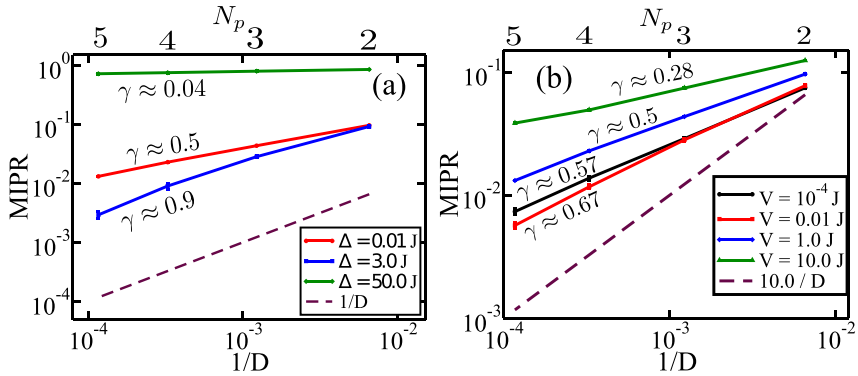

FIG. 16. The log-log plot of the spectrum-averaged MIPR with $1 / D$ [where $D=\left(\begin{array}{c}N \\ N_{p}\end{array}\right)$ ], keeping the system size $N=18$ and increasing the number of fermions from $N_{p}=2,3,4$, and 5 for (a) fixed interaction strength $V=1.0 J$ and different disorder strength $\Delta$ and (b) for fixed $\Delta=0.01 J$ and different interaction strength $V$. The dashed line shows a linear relationship between MIPR and $1 / D$.

Effect offilling fraction. First we explore the effect of filling fraction $v$ by looking at the scaling relation MIPR $\propto \frac{1}{D^{\gamma}}$. For a perfectly delocalized many-body phase $\gamma=1$ whereas for a nonergodic many-body phase $0<\gamma<1$. Deep in the nonergodic MBL phase $\gamma$ can be as small as close to 0 [48]. Fixing $V=1.0 \mathrm{~J}$, we show the MIPR as a function of $1 / D$ for different disorder strengths in Fig. 16(a). The scaling analysis shown in the figure indicates that, as filling fraction increases, the many-body system is in the MBL phase for large $\Delta$ and is delocalized in the intermediate range of $\Delta$. In the low $\Delta$ regime, the system is a mixed one with $\gamma=0.5$. To explore the behavior of the mixed phase, in Fig. 16(b), we plot MIPR versus $1 / D$ for different $V$ by fixing $\Delta=0.01 J$. The increasing values of $\gamma$ with respect to interaction indicates that, in a finite system, this phase is a mixed one and the amount of delocalization is high (red curve) when $V \approx \Delta$. For $V=10.0 \mathrm{~J}$ in Fig. 16(b), the value of $\gamma$ is much smaller due to the strong repulsive interaction aiding localization.

Effect of system size. In Fig. 17, we show the spectrum averaged MIPR as a function of disorder strength $\Delta$ for system sizes $N=12,18$, and 24 and for filling fraction $v=1 / 6$, and interaction strength $V=1.0 \mathrm{~J}$. MIPR decreases rapidly with $N$ for the intermediate values of $\Delta$ indicating a delocalization in the many-body system. The trend of the plots suggests that the many-body system may thermalize for intermediate values of $\Delta$ as $N$ increases. However, for large $\Delta$, MIPR barely changes implying a possible many-body
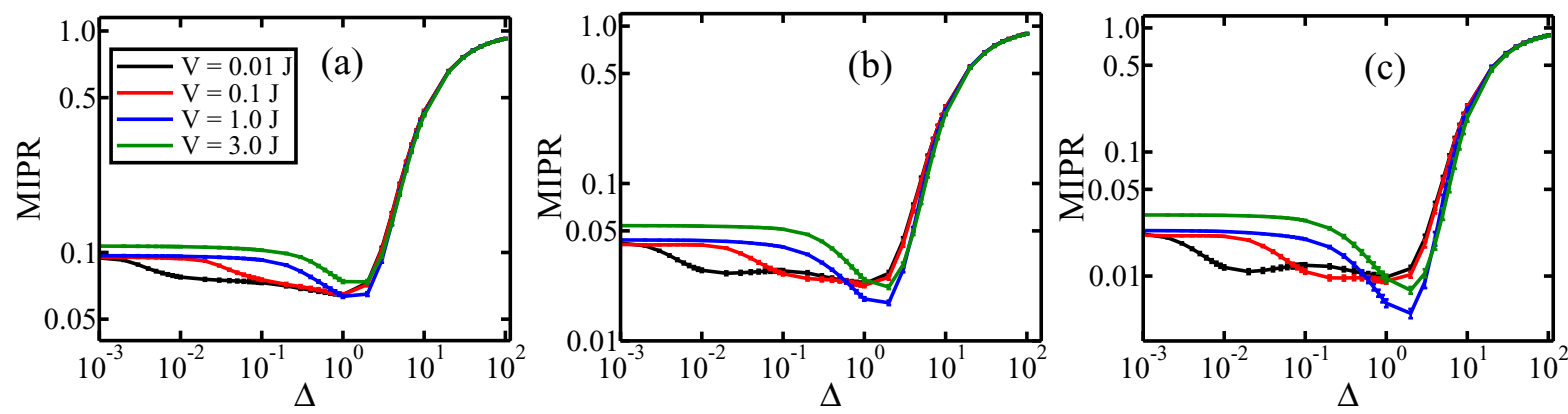

FIG. 15. The log-log plots of the spectrum-averaged MIPR as a function of $\Delta$ (in units of $J$ ) for different interaction strengths $V$ for fermionic filling fraction (a) $1 / 9$, (b) $1 / 6$, and (c) $2 / 9$, respectively. For all the plots system size, $N=18$. Number of disorder realizations are 500,200 and 100 for $1 / 9,1 / 6$, and $2 / 9$ fillings, respectively. 


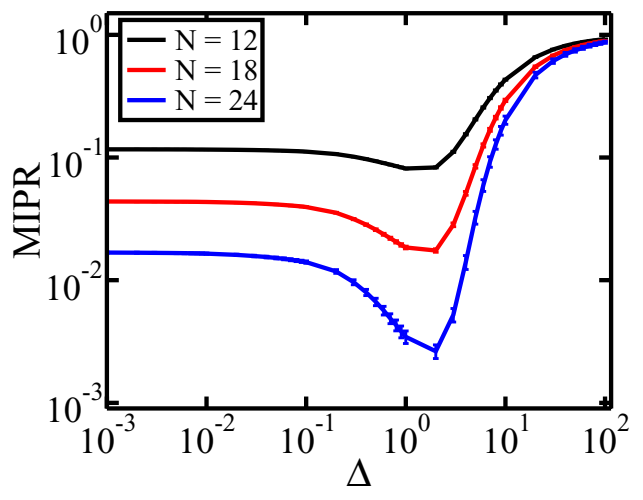

FIG. 17. The spectrum averaged MIPR as a function of disorder strength $\Delta$ (in units of $J$ ) for fixed $V=1.0 J$ and system sizes $N=12$, 18 , and 24 for filling fraction of fermions $v=1 / 6$. The quantities are averaged over 500, 200, and 30 disorder realizations for $N=12,18$, and 24, respectively, for fermion filling $v=1 / 6$.

localization in the system. In the small $\Delta$ regime, MIPR decreases with increase in system size, but not as fast as compared to the intermediate $\Delta$ regime. This implies that for the small disorder strengths $\Delta$, the system does not attain thermalizaion and appears to be in a distinct nonergodic phase as the system size increases. Next we analyze the effect of system size $N$ in the low-disorder phase. To explore the characteristics of the phase at low disorder strengths, we fix $\Delta=0.01 J$, and plot MIPR vs $1 / D$ for different $V$ in Fig. 18 . The plots affirm the conclusions made from Fig. 16(b); the values of $\gamma$ indicate that the phase is neither thermal nor MBL and hence it is dubbed as the mixed phase. The amount of delocalization in the mixed phase increases when $V=\Delta$. For $V=1.0 \mathrm{~J}, \gamma=0.38$ implying localization-dominated mixed phase. What happens to the fate of the mixed phase in the thermodynamic limit may need a further study which goes beyond exact diagonalization.

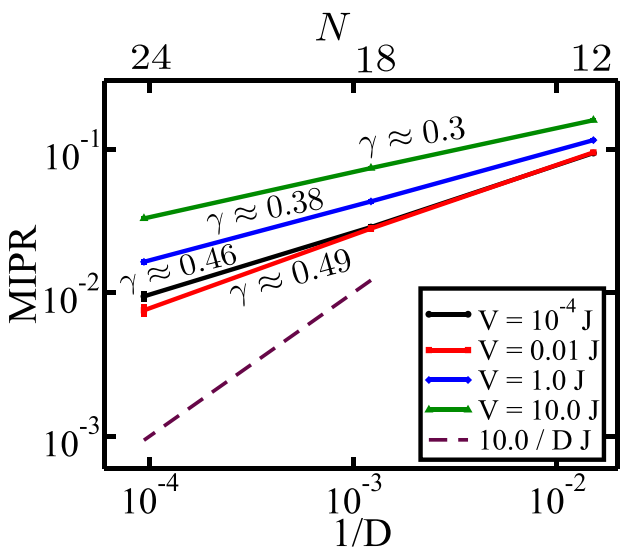

FIG. 18. The log-log plot of the spectrum-averaged MIPR with $1 / D$ [where $D=\left(\begin{array}{c}N \\ N_{p}\end{array}\right)$ ], keeping the system filling fraction $v=1 / 6$ and increasing the system size from $N=12,18$, and 24 for fixed $\Delta=0.01 \mathrm{~J}$ and increasing interaction strength $V$. The dashed line shows a linear relationship between MIPR and $1 / D$.
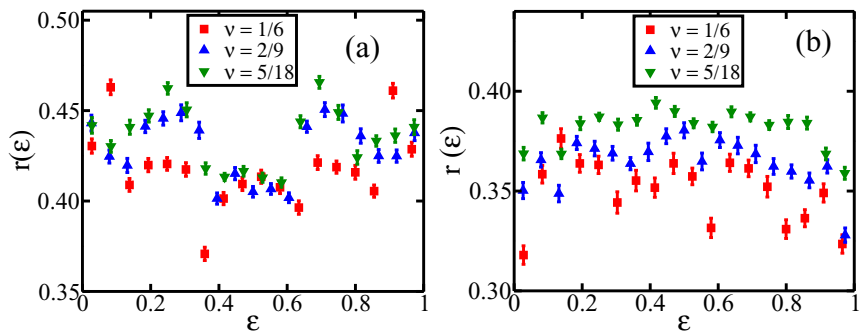

FIG. 19. (a) The energy resolved $r(\varepsilon)$ for increasing values of filling fraction $v=1 / 6,2 / 9$, and $5 / 18$ for $N=18$ and $V=\Delta=$ $0.01 \mathrm{~J}$. (b) Similar plots but for $V=1.0 \mathrm{~J}$ and $\Delta=0.01 \mathrm{~J}$. For all the plots, number of disorder realizations is 200 .

\section{Energy-resolved level statistics, inverse participation ratio and entanglement entropy}

Here we carry out an energy-resolved study of the levelspacing ratio, many-body inverse participation ratio and entanglement entropy to get some further insights into the system, especially when it is in the mixed phase. We divide the many-body energy spectrum into a number of equal segments and calculate the local average of the quantities for each segment of the spectrum. We denote the energy resolved quantities as $r(\varepsilon), \operatorname{MIPR}(\varepsilon)$, and $S_{A}(\varepsilon)$ respectively, where $\varepsilon$ 's are the fractional eigenstate index at the middle of each segment.

We first discuss the energy resolved level-spacing ratio $r(\varepsilon)$ in the small disorder $\Delta \ll J$ regime. In Fig. 19(a), we show $r(\varepsilon)$ for increasing filling fraction $v$ for $V=\Delta=0.01 \mathrm{~J}$. With increasing $v, r(\varepsilon)$ increases and shifts away from 0.386 implying more delocalization for $V=\Delta$ as predicted by other analysis done in the paper. In Fig. 19(b), $r(\varepsilon)$ for increasing filling fraction $v$ is shown for $V=1.0 \mathrm{~J}$ and $\Delta=0.01 \mathrm{~J}$. Here, with increasing $v, r(\varepsilon)$ approaches 0.386 except at the edges of the spectrum. This is a signature of localization in the system. However from the MIPR $\propto 1 / D^{\gamma}$ scaling one concludes that it is a mixed phase as $\gamma=0.5$. We also show $r(\varepsilon)$ with increasing system size $N$ for fixed $\Delta=0.01 \mathrm{~J}$ and $V=0.01 J$ and $1.0 J$ in Figs. 20(a) and 20(b), respectively. When $V=\Delta=0.01 J, r(\varepsilon)$ increases from 0.386 indicating a delocalizing effect in the system. When $V=1.0 \mathrm{~J}$ and $\Delta=$ $0.01 J, r(\varepsilon)$ is consistently spread around 0.386 for $N=24$, indicating an effective localization in the system. Also from MIPR scaling we find $\gamma=0.38$ implying the dominance of
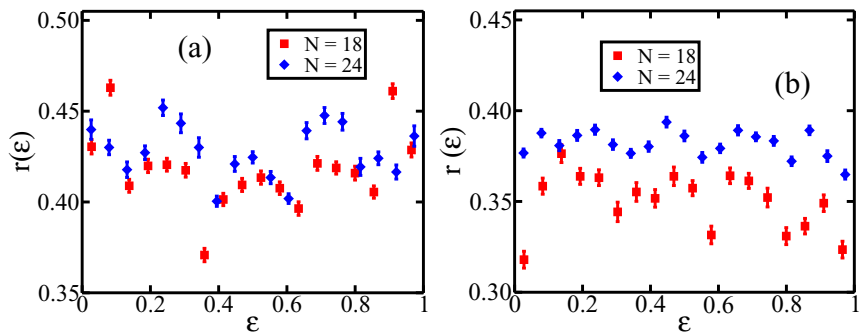

FIG. 20. (a) The energy resolved $r(\varepsilon)$ for increasing values of system size $N=18$ and 24 for $v=1 / 6$ and $V=\Delta=0.01 J$. (b) Similar plots but for $V=1.0 \mathrm{~J}$ and $\Delta=0.01 \mathrm{~J}$. For all the plots, number of disorder realizations is 200 . 


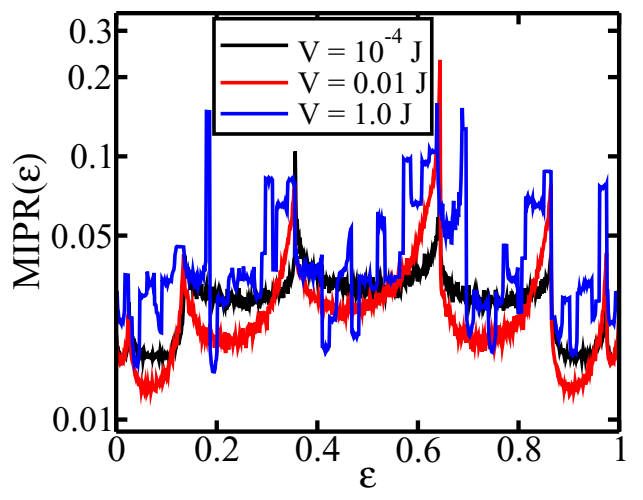

FIG. 21. The MIPR $(\varepsilon)$ of all the eigenstates as a function of the fractional eigenstate index $\varepsilon$ for $\Delta=0.01 J$ and different interaction strengths $V$. For all the plots, $N=18, v=1 / 6$, and the number of disorder realizations is 200 .

the localized eigenstates. Finite-size effects are rather severe here and make it difficult to make a conclusive statement on whether the system will actually become many-body localized in the thermodynamic limit.

The MIPR of all the many-body eigenstates is shown in Fig. 21 for $\Delta=0.01 \mathrm{~J}$ and different interaction strengths $V$, while keeping $N=18$ and $v=1 / 6$. The figure captures the localization properties of all the eigenstates in the small $\Delta$ regime. We can see that for all values of $V$, the MIPR of all the eigenstates are significantly higher than $1 / D$ but not close to 1 implying that the system is in a mixed phase. Although when $V=\Delta$, the majority of the eigenstates show delocalization tendency that amounts to an overall delocalizing effect in the system. As $V>\Delta$ many of the eigenstates show high values of MIPR with occasional presence of the low-MIPR eigenstates. This keeps the system still in a mixed phase with a greater proportion of localization.

As an accompanying quantity the half-chain entanglement entropy $S_{A}$ of all the many-body eigenstates is shown in Fig. 22(a) for $\Delta=0.01 \mathrm{~J}$ and different interaction strengths $V$, while once again keeping $N=18$ and $v=1 / 6$ fixed. It is noticeable that when $V \ll \Delta$ there are eigenstates with high and occasional low entanglement entropies. Although when $V=\Delta$, the majority of the eigenstates has significantly high entanglement entropy whereas the few occasional
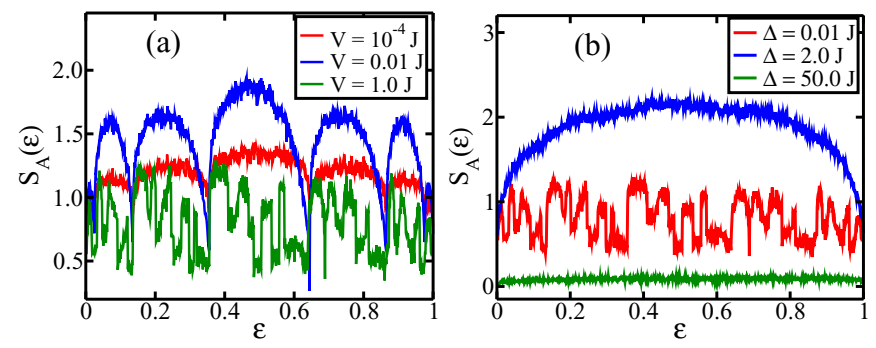

FIG. 22. The half-chain entanglement entropy $S_{A}(\varepsilon)$ of all the eigenstates as a function of the fractional eigenstate index $\varepsilon$ (a) for increasing values of $V$ and fixed $\Delta=0.01 J$ and (b) for increasing values of $\Delta$ and fixed $V=1.0 \mathrm{~J}$. For all the plots, $N=18, v=1 / 6$, and number of disorder realizations is 200 .
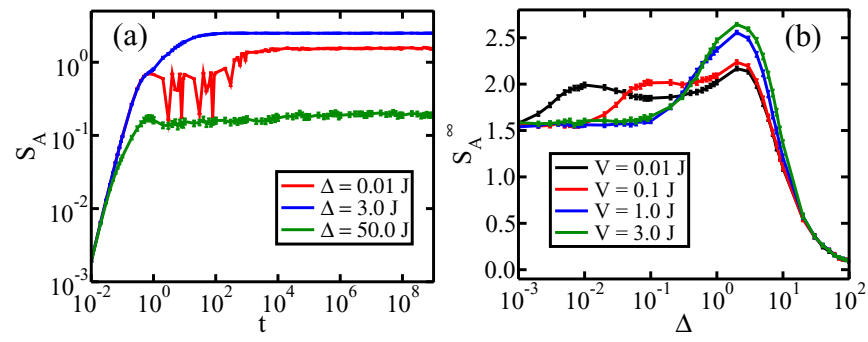

FIG. 23. (a) The half-chain entanglement entropy $S_{A}$ as a function of time $t$ (in units of $J^{-1}$ ) for $N=18, V=1.0 J$, and $\Delta=$ $0.01 \mathrm{~J}, 3.0 \mathrm{~J}$, and 50.0J. (b) The saturation value $S_{A}^{\infty}$ as a function of $\Delta$ (in units of $J$ ) for increasing values of $V$ and $N=18$. For all the plots, number of disorder realizations is 200 .

eigenstates have significantly low entanglement entropies, indicating dominance of delocalization in the mixed phase. As $V>\Delta$ majority of the eigenstates shows low entanglement entropy implying a dominance of localization. In Fig. 22(b), half-chain $S_{A}$ of all the eigenstates are shown for interaction $V=1.0 \mathrm{~J}$ and $\Delta=0.01 \mathrm{~J}, 2.0 \mathrm{~J}, 50.0 \mathrm{~J}$ such that the system is in the mixed, thermal and MBL phases respectively. As can be seen from the figure, the thermal and MBL phases give rise to a smooth dependence of $S_{A}$ on eigenstates with very high and very low $S_{A}$, respectively. In the mixed phase, eigenstate entanglement entropies have many humps and dips of intermediate strength.

\section{Nonequilibrium dynamics of interacting spinless fermions}

In this section, we discuss many-body nonequilibrium dynamics, keeping track of the entanglement entropy, the return probability and imbalance parameter. We choose the initial state as an experimentally relevant, density-wave type of state as mentioned previously $\left|\Psi_{i n}\right\rangle=\prod_{i=1}^{N / 3} \hat{c}_{2 i}^{\dagger}|0\rangle$, which is a product state with the filling fraction of fermions $v=1 / 6$ with $N=18$. The dynamics of the entanglement entropy $S_{A}$ (the subsystem $\mathrm{A}$ is the first half of the full system) is calculated via exact diagonalization for $V=1.0$ and is shown in Fig. 23(a) for different disorder strengths. For low $\Delta=0.01 \mathrm{~J}$ after a super-ballistic transient, there is a damped oscillatory behavior (see Appendix A for zero-disorder case) followed by a substantially sub-diffusive $\left(t^{0.05}\right)$ regime till $S_{A}$ reaches saturation. The little post-oscillatory increment in $S_{A}$ is the effect of nonzero interaction $V$ leading to a mixed phase with a small amount of delocalization in the system. For intermediate disorder $\Delta=3.0 \mathrm{~J}$, after the transient, there is a sub-diffusive $\left(t^{0.3}\right)$ increment in $S_{A}$ before it quickly saturates to a large value reflecting delocalization in the many-body system. The oscillatory part is absent in this case. For large $\Delta=50.0 \mathrm{~J}$, after the transient, $S_{A}$ saturates to a lower value indicating many-body localization. A finer analysis of the dynamical behavior of the MBL system is given in Appendix C. The dependence of the saturation value of the entanglement entropy $S_{A}^{\infty}$ on disorder strength $\Delta$ for $N=18, v=1 / 6$, and different $V$ is shown in Fig. 23(b). Typically the entanglement entropy in the many-body delocalized phase is substantially higher as compared to the same in the MBL phase. For $V \approx J$, we observe the presence of three phases: MBL for large $\Delta$, 

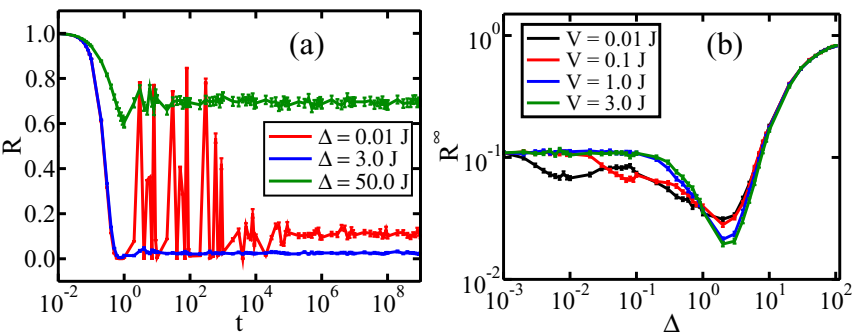

FIG. 24. (a) The return probability $R$ as a function of time $t$ (in units of $J^{-1}$ ) for $N=18, V=1.0 \mathrm{~J}$, and $\Delta=$ $0.01 \mathrm{~J}, 3.0 \mathrm{~J}$, and 50.0J. (b) The saturation value of the return probability $R^{\infty}$ as a function of $\Delta$ (in units of $J$ ) for different values of $V$ and $N=18$. The number of disorder realizations is 200 for both the plots.

delocalization for intermediate $\Delta$ and the mixed phase for small $\Delta$. For $V<J$ another signature of delocalization is noted when $V \approx \Delta$. All these results are consistent with the results of MIPR and $r$ as discussed in the previous subsection. Plots similar to Fig. 23(b) are also obtained for the spectrum averaged entanglement entropy (Fig. 27 in Appendix B).

Although entanglement entropy offers useful insights, it is not easily measurable in ongoing experiments. In this context, return probability and imbalance parameter, being experimentally accessible, are interesting and we study them next. The return probability is defined as $R(t)=\left|\left\langle\Psi_{i n} \mid \Psi_{t}\right\rangle\right|^{2}$. In the perfectly delocalized phase $R=1 / D$ whereas in the perfectly localized phase $R$ is unity. In Fig. 24(a), the dynamics of $R$ is shown for $V=1.0 \mathrm{~J}$ for different $\Delta$. For $\Delta=0.01 \mathrm{~J}$, just like $S_{A}, R$ also shows oscillatory behavior indicating recurrence before it saturates to a finite value. For $\Delta=3.0 \mathrm{~J}$, the oscillatory behavior is absent and the saturation value is sufficiently low indicating delocalization. For $\Delta=50.0 J, R$ saturates to a significantly higher value indicating strong localization. The saturation value $R^{\infty}$ versus $\Delta$ plots for increasing values of $V$ are shown in Fig. 24(b). From this figure one can infer the presence of three phases: MBL for large $\Delta$, delocalization for intermediate $\Delta$ and the mixed phase for small $\Delta$. For $V \ll J$, another signature of delocalization is noted when $V \approx \Delta: R^{\infty}$ shows dips at those points, but these dips are not as deep as those corresponding to $V \approx J$.

The imbalance parameter $I_{b}$ is already defined in Eq. (11) in the previous section. In the thermalized and MBL phases respectively, we expect the saturation values to be $I_{b}=-2 / 3$ and $I_{b}=1$ respectively. In Fig. 25(a), the dynamics of $I_{b}$ is shown for $V=1.0$ and different $\Delta$. For $\Delta=0.01 J, I_{b}$ saturates to -0.19 , which is higher than $-2 / 3$, after an oscillatory behavior due to recurrence. For $\Delta=3.0 J$, the recurrence is absent and the saturation value is low indicating delocalization whereas for $\Delta=50.0 \mathrm{~J}, I_{b}$ saturates to a much higher value implying localization in the many-body system. The saturation value $I_{b}^{\infty}$ versus $\Delta$ plots for different $V$ are shown in Fig. 25(b). Similar to $S_{A}^{\infty}$ and $R^{\infty}, I_{b}^{\infty}$ confirms the presence

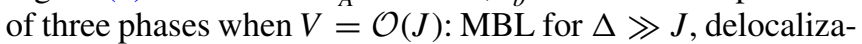
tion for $\Delta \approx J$ and the mixed phase for $\Delta \ll J$. For $V \ll J$, an increment in the amount of delocalization is notable in the mixed phase when $V \approx \Delta$ as small dips are visible at those points. To reaffirm our findings, we have studied the
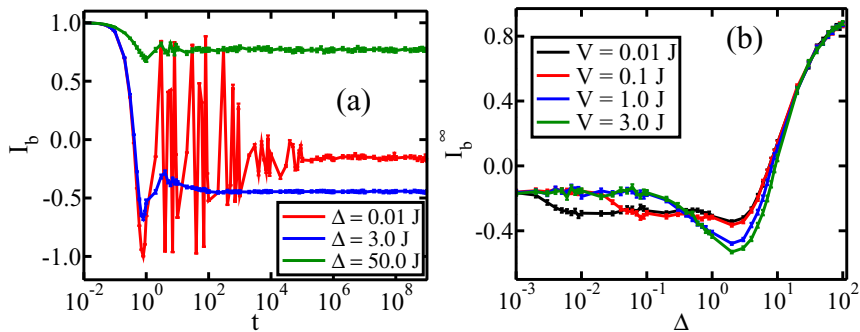

FIG. 25. (a) The imbalance parameter $I_{b}$ as a function of time $t$ (in units of $J^{-1}$ ) for $N=18, V=1.0 \mathrm{~J}$ and $\Delta=0.01 \mathrm{~J}, 3.0 \mathrm{~J}, 50.0 \mathrm{~J}$. (b) The saturation value $I_{b}^{\infty}$ as a function of $\Delta$ (in units of $J$ ) for different values of $V$ and $N=18$. For all the plots, number of disorder realizations is 200 .

system size dependence of the time-evolved many body state in the long-time limit (Appendix D), which also indicates the presence of three distinct phases as observed in the study of imbalance parameter.

\section{CONCLUSIONS}

We report a systematic investigation of the effect of disorder and interactions on a flat-band supporting diamond chain. Disorder detunes and hybridizes the compact localized states associated with flat bands and as the strength of disorder is increased, flat-band based localization and conventional Anderson localization are observed. Observables obtained from both eigenvalues and eigenfunctions shine light into the distinct features of the different phases. We observe a hierarchy of localization: compact localization $>$ Anderson localization > flat-band based localization.

Single-particle wave-packet dynamics complements the results obtained via statics. In the disorder-free limit, a persisting oscillatory recurrence which is attributed to the compact localized states is observed. A damped oscillatory recurrence is observed in the flat-band based localization phase and no oscillatory recurrence is observed in the Anderson localization phase. The on-site occupation probability of the single particle in the long-time limit confirms that the localization strength can be classified as compact localization $>$ Anderson localization > flat-band based localization. We note that in other all band flat lattices like one-dimensional cross-stitch lattice [49] and two-dimensional dice lattice [28] the same hierarchy is maintained.

Noninteracting many-particle fermion states also show characteristics of flat-band based localization and Anderson localization in the presence of low and high disorder strengths. A study of entanglement entropy and imbalance reveals a delocalizing tendency for intermediate disorder strengths, reflecting the effect of fermionic statistics in the system. The low-disorder and high-disorder phases are distinguished by the presence and absence of the characteristic damped oscillatory recurrence in the nonequilibrium dynamics of the entanglement entropy and imbalance.

The interplay of disorder and interaction in a finite system can lead to three distinct phases: MBL for high disorder strength, thermal phase for intermediate disorder strength and nonergodic mixed phase for low disorder strengths. We 
observe that, in the mixed phase, the eigenstates tend to get delocalized when interaction strength is of the order of disorder-strength. A detailed analysis of the scaling of the spectrum-averaged many-body inverse participation ratio with the dimension of the Hilbert space is carried out by varying the filling fraction and system size. We observe that at higher filling fractions, the mixed phase in the finite system tends to have more dominance of delocalization as long as interaction strength is less than the hopping strength. However, an increment of localization in the mixed phase is observed for high enough interaction-strength. When the interaction strength is comparable to the hopping strength, for the intermediate and higher ranges of disorder-strength, one obtains the crossovers from the nonergodic mixed-to thermal and thermal-to-MBL phases respectively, which become more distinct as the filling fraction and system size increase. Nonequilibrium dynamics supports the main findings from the above study involving statics. A characteristic damped oscillatory behavior is found in the mixed phase whereas no such oscillations are found in the thermalized and MBL phases. In order to comment on the thermodynamic existence of the three phases, especially on the fate of the mixed phase, one needs a further study which is not limited by the Hilbert-space constraint of numerical exact diagonalization. We think that our work will help motivate such studies on the diamond chain in the future.

The joint presence of disorder and interactions in flat-band based systems give rise to rich and unconventional phases obtained both from single-particle and many-particle properties of the system. These phases could potentially be realized in cold-atom based experiments. This kind of a systematic study of flat-band supporting systems is lacking in the literature. We believe that our work will trigger more work on flat-band based systems.

\section{ACKNOWLEDGMENTS}

We are grateful to the High Performance Computing (HPC) facility at IISER Bhopal, where large-scale computations of this project were run. N.R is grateful to the University Grants Commission (UGC), India for his Ph.D fellowship. A.S is grateful to SERB for the grant (File No. CRG/2019/003447), and the DST-INSPIRE Faculty Award [DST/INSPIRE/04/2014/002461].

\section{APPENDIX A: OSCILLATORY DYNAMICS OF THE REVIVAL PROBABILITY: SINGLE PARTICLE, NONINTERACTING, AND INTERACTING FERMIONS}

Here we show the results for the dynamics of the revival probability of a single particle, for noninteracting fermions, and for interacting fermions in the clean system. We also compare results for the clean system with those for the system in the low-disorder regime. The zero-disorder $(\Delta=0.0)$ dynamics of the revival probability $R$ is shown in Figs. 26(a), 26(b) and $26(\mathrm{c})$ for a single particle, noninteracting fermions, and interacting fermions, respectively. It is interesting to see that $R$ is oscillatory both in real space and in the particle-number constrained Hilbert space. This oscillation never stops and can be observed in the other dynamical quantities (such as calculated in the main text) as well. Similar recurring behavior has been reported in a study of interacting bosons in the clean diamond chain [50]. The full dynamics of $R$ for $\Delta=0$ is compared with that for small $\Delta=0.01 \mathrm{~J}$ for a single particle, noninteracting fermions, and interacting fermions in Fig. 26(d), 26(e), and 26(f), respectively. However the oscillatory behavior is not visible in these figures due to the variable step size in time and hence lack of data points. For $\Delta=0.01 \mathrm{~J}$,
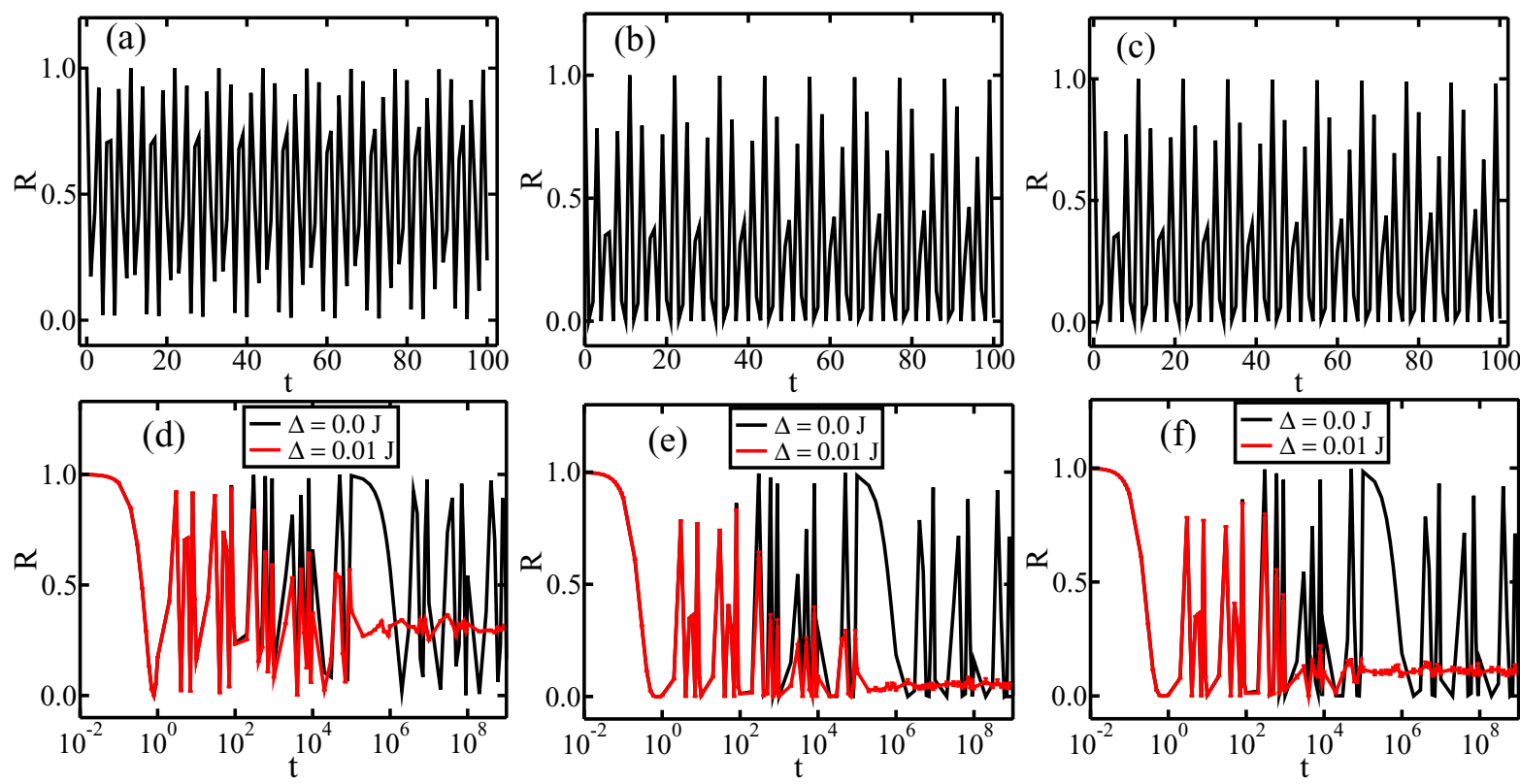

FIG. 26. [(a)-(c)] The oscillatory behavior of the revival probability $R$ as a function of time (in units of $J^{-1}$ ) for a single particle, noninteracting fermions, and interacting fermions respectively for $\Delta=0.0$. [(d)-(f)] The full dynamics of $R$ for $\Delta=0.0$ is compared to the that for small $\Delta=0.01 \mathrm{~J}$ for a single particle, noninteracting fermions and interacting fermions respectively. Here, the time axis is in a logarithmic scale. For a single particle, $N=600$, whereas for both noninteracting and interacting fermions, $N=18$ with filling of fermions $v=1 / 6$. Interaction strength $V=1.0 \mathrm{~J}$ for interacting fermions. 


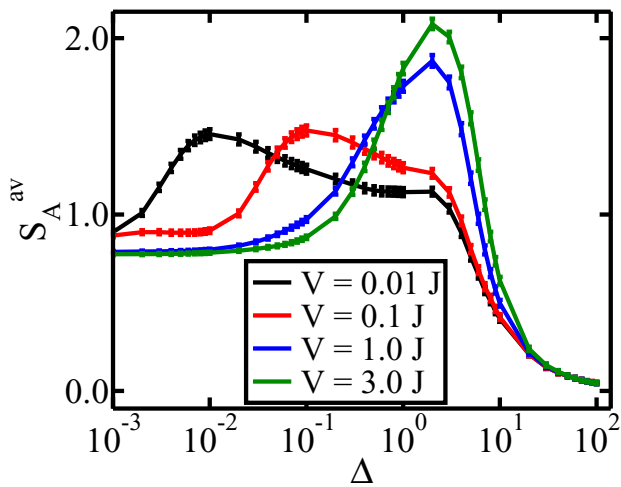

FIG. 27. The spectrum averaged entanglement entropy $S_{A}^{\mathrm{av}}$ as a function of disorder strength $\Delta$ (in units of $J$ ) for increasing values of $V$ for $N=18$ and $v=1 / 6$. For all the plots, number of disorder realizations is 100 .

the amplitude of the oscillation keeps decreasing and vanishes at some point as $R$ saturates to a finite value for all three cases.

\section{APPENDIX B: SPECTRUM-AVERAGED ENTANGLEMENT ENTROPY FOR THE INTERACTING SYSTEM}

Here, we discuss the half-chain entanglement entropy $S_{A}^{\mathrm{av}}$, averaged over all the eigenstates. It is a useful quantity to help characterize different many-body phases in the presence of disorder $\Delta$ and interaction strength $V$. In Fig. 27, the $S_{A}^{\text {av }}$ versus $\Delta$ plots are shown for increasing values of $V$ for $N=18$ and $v=1 / 6$. In the delocalized phase, $S_{A}^{\mathrm{av}}$ should attain a higher value as compared to that for the localized phase. From the figure, we see the presence of three phases: MBL phase for $\Delta \gg J$, mixed phase for $\Delta \ll J$ and delocalized phase for intermediate $\Delta=\mathcal{O}(J)$ when $V=\mathcal{O}(J)$. For $\Delta \ll J$, a delocalizing effect is found when $V \approx \Delta$. All these results complement the results obtained from MIPR and $r$ as discussed in the main text. Also the plots of $S_{A}^{\mathrm{av}}$ resemble the plots in Fig. 23(b).

\section{APPENDIX C: FURTHER INSIGHTS INTO THE ENTANGLEMENT GROWTH IN THE MBL PHASE}

We fit the curve representing the growth of entanglement entropy $S_{A}$ from an initial product state of the system in the MBL phase. The parameters $\Delta=50.0 \mathrm{~J}, V=1.0 \mathrm{~J}, N=18$, and $v=1 / 6$ are chosen in the regime where the level-spacing ratio $r \approx 0.386$ establishing the MBL phase. For a system of the spinless fermions with nearest-neighbor interactions in a one-dimensional linear chain, $S_{A}$ grows logarithmically with time in the MBL phase [51,52]. Here we have obtained the time dependence of $S_{A}$ for the system in the quasi onedimensional diamond chain. From Fig. 28(a), the growth of $S_{A}$ is fitted with a logarithmic function $y=a \ln (x)+b$ with $a=0.00447 ; b=0.12569$. One cannot rule out the possibility of obtaining a more-than-logarithmic dependence for systems having higher dimensions. We also attempted a power-law fit for the same data [Fig. 28(b)]: $y=a x^{b}$ with $a=0.13022$ and $b=0.02650$ with the power-law exponent being tiny. Recently in Refs. $[53,54]$ the possibility of MBL in

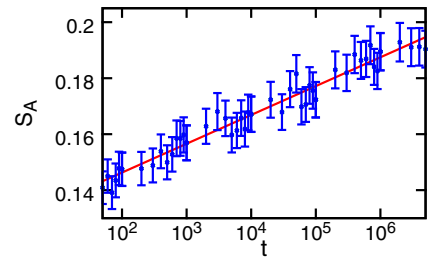

(a)

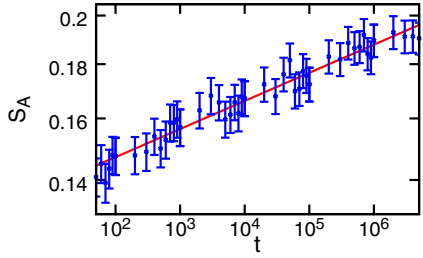

(b)
FIG. 28. Plots of entanglement entropy $\left(S_{A}\right)$ growth with time (in units of $J^{-1}$ ) in the MBL phase, fitted with (a) $y=a \ln (x)+b$ with $a=0.00447 ; b=0.12569$ and (b) $y=a x^{b}$ with $a=0.13022 ;, b=$ 0.02650 . Fitted curves are shown in solid red lines whereas the original data-points are shown in blue color. (a) is a semilogarithmic plot whereas figure (b) is a log-log plot. For all the plots, the disorder strength $\Delta=50.0 \mathrm{~J}$, interaction strength $V=1.0 \mathrm{~J}$ for $N=18$ and $v=1 / 6$ with number of disorder realizations used being 1000 .

quasi-1D has been indicated. This Appendix is an attempt towards investigating the dynamical signatures of the quasi-1D MBL. While our results are certainly consistent with MBL, much larger system sizes would be required to conclusively address the question of whether logarithmic or power-law behavior holds. The consequences of the simultaneous presence of interaction and higher-dimensionality effects deserve a thorough separate investigation.

\section{APPENDIX D: NORMALIZED PARTICIPATION RATIO}

The dependence of time evolved quantum many-body state on system size in the long-time limit has turned out to be handy to decipher many-body phases [55]. In this section, we stick to the filling fraction $v=1 / 3$ and consider the initial state $\left|\Psi_{i n}\right\rangle=\prod_{i=1}^{N / 3} \hat{c}_{i}^{\dagger}|0\rangle$. Any time evolved state can then be generically written as $|\Psi(t)\rangle=\sum_{n=1}^{D} C_{n}(t)|n\rangle$, where $|n\rangle$ stands for the $n$th configuration and $D$ is the size of the Hilbert space. The normalized participation ratio $(N P R)$ of the manybody state in the long-time limit $(t \rightarrow \infty)$ is defined as [55]

$$
\eta=\frac{1}{D \sum_{n}\left|C_{n}(t \rightarrow \infty)\right|^{4}} .
$$

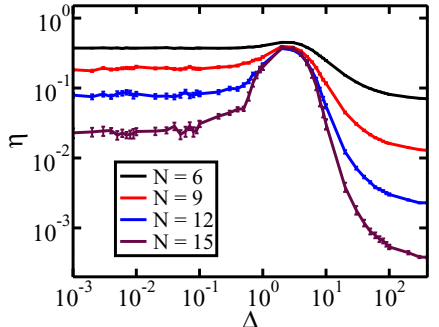

(a)

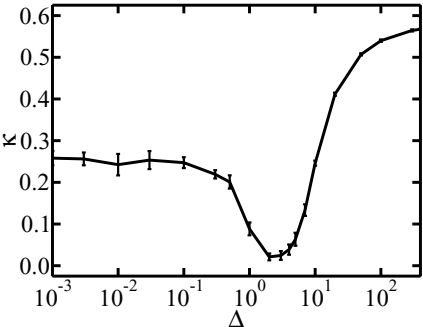

(b)
FIG. 29. The scaling analysis of satuaration of NPR. (a) A log$\log$ plot showing the values of $\eta$ as a function of disorder strength $\Delta$ (in units of $J$ ) for increasing system size $N$. (b) A semi-log plot of the scaling exponent $\kappa$ as a function of $\Delta$ (in units of $J$ ). For all the plots the interaction strength $V=1.0 \mathrm{~J}$ and filling fraction $v=1 / 3$. The number of disorder realizations varies for different system sizes, but all of them have at least 100 samples of disorder. 
In the ergodic phase phase, $\eta$ must be independent of system size $N$ whereas in the nonergodic phase, $\eta$ must depend on $N$. It has been shown that $\eta \propto e^{-\kappa N}$ with $\kappa \approx 0.5$ for the many-body localized phase in a quasiperiodic chain [55].

In Fig. 29(a), we show the dependence of $\kappa$ on disorder strength $\Delta$ for fixed interaction strength $V=1.0 \mathrm{~J}$ and increasing system size $N$. In the large and small disorder regimes, $\eta$ changes with system size, while for the intermediate range of $\Delta, \eta$ seems to be system size independent. For each value of $\Delta$, the exponent $\kappa$ can be extracted using the relation $\eta \propto e^{-\kappa N}$. The exponent $\kappa$ as a function of $\Delta$ is shown in Fig. 29(b). In the large $\Delta$ regime, $\kappa \approx 0.5$ implying the nonergodic many-body localized phase whereas for intermediate values of $\Delta, \kappa \approx 0$ implying the ergodic phase. In the small $\Delta$ regime $\kappa \approx 0.25$, which definitely is a sign of nonergodicity. However, since $\kappa$ is not as high as 0.5 this mixed phase may not be as nonergodic as the MBL. In addition, we have verified that the scaling analysis of the spectrum averaged NPR in the static case leads to similar results as shown in Fig. 29. Also, the analyses reported in the main text point towards a similar conclusion. The low-disordered nonergodic phase could be a remnant of a new kind of MBL phase recently found in the clean limit of such systems [34].
[1] S. Flach, D. Leykam, J. D. Bodyfelt, P. Matthies, and A. S. Desyatnikov, Detangling flat bands into fano lattices, Europhys. Lett. 105, 30001 (2014).

[2] L. Toikka and A. Andreanov, Necessary and sufficient conditions for flat bands in m-dimensional n-band lattices with complex-valued nearest-neighbour hopping, J. Phys. A: Math. Theor. 52, 02 LT04 (2018).

[3] O. Derzhko, J. Richter, and M. Maksymenko, Strongly correlated flat-band systems: The route from heisenberg spins to hubbard electrons, Int. J. Mod. Phys. B 29, 1530007 (2015).

[4] W. Maimaiti, A. Andreanov, H. C. Park, O. Gendelman, and S. Flach, Compact localized states and flat-band generators in one dimension, Phys. Rev. B 95, 115135 (2017).

[5] A. Ramachandran, A. Andreanov, and S. Flach, Chiral flat bands: Existence, engineering, and stability, Phys. Rev. B 96, 161104(R) (2017)

[6] S. Mukherjee and R. R. Thomson, Observation of localized flat-band modes in a quasi-one-dimensional photonic rhombic lattice, Opt. Lett. 40, 5443 (2015).

[7] E. Travkin, F. Diebel, and C. Denz, Compact flat band states in optically induced flatland photonic lattices, Appl. Phys. Lett. 111, 011104 (2017).

[8] S. Klembt, T. H. Harder, O. A. Egorov, K. Winkler, H. Suchomel, J. Beierlein, M. Emmerling, C. Schneider, and S. Höfling, Polariton condensation in s-and p-flatbands in a twodimensional lieb lattice, Appl. Phys. Lett. 111, 231102 (2017).

[9] O. Derzhko, J. Richter, A. Honecker, M. Maksymenko, and R. Moessner, Low-temperature properties of the hubbard model on highly frustrated one-dimensional lattices, Phys. Rev. B 81, 014421 (2010).

[10] H. Takeda, T. Takashima, and K. Yoshino, Flat photonic bands in two-dimensional photonic crystals with kagome lattices, J. Phys.: Condens. Matter 16, 6317 (2004).

[11] M. Goda, S. Nishino, and H. Matsuda, Inverse Anderson Transition Caused by Flatbands, Phys. Rev. Lett. 96, 126401 (2006).

[12] G.-B. Jo, J. Guzman, C. K. Thomas, P. Hosur, A. Vishwanath, and D. M. Stamper-Kurn, Ultracold Atoms in a Tunable Optical Kagome Lattice, Phys. Rev. Lett. 108, 045305 (2012).

[13] S. Taie, H. Ozawa, T. Ichinose, T. Nishio, S. Nakajima, and Y. Takahashi, Coherent driving and freezing of bosonic matter wave in an optical lieb lattice, Sci. Adv. 1, e1500854 (2015).

[14] R. A. Vicencio, C. Cantillano, L. Morales-Inostroza, B. Real, C. Mejía-Cortés, S. Weimann, A. Szameit, and M. I. Molina,
Observation of Localized States in Lieb Photonic Lattices, Phys. Rev. Lett. 114, 245503 (2015).

[15] S. Mukherjee, A. Spracklen, D. Choudhury, N. Goldman, P. Öhberg, E. Andersson, and R. R. Thomson, Observation of a Localized Flat-Band State in a Photonic Lieb Lattice, Phys. Rev. Lett. 114, 245504 (2015).

[16] S. Mukherjee, M. Di Liberto, P. Öhberg, R. R. Thomson, and N. Goldman, Experimental Observation of Aharonov-Bohm Cages in Photonic Lattices, Phys. Rev. Lett. 121, 075502 (2018).

[17] N. Masumoto, N. Y. Kim, T. Byrnes, K. Kusudo, A. Löffler, S. Höfling, A. Forchel, and Y. Yamamoto, Exciton-polariton condensates with flat bands in a two-dimensional kagome lattice, New J. Phys. 14, 065002 (2012).

[18] B. Pal, Nontrivial topological flat bands in a diamond-octagon lattice geometry, Phys. Rev. B 98, 245116 (2018).

[19] K. Zegadlo, N. Dror, N. Viet Hung, M. Trippenbach, and B. A. Malomed, Single and double linear and nonlinear flatband chains: Spectra and modes, Phys. Rev. E 96, 012204 (2017).

[20] P. Shukla, Disorder perturbed flat bands: Level density and inverse participation ratio, Phys. Rev. B 98, 054206 (2018).

[21] J. D. Bodyfelt, D. Leykam, C. Danieli, X. Yu, and S. Flach, Flatbands Under Correlated Perturbations, Phys. Rev. Lett. 113 236403 (2014).

[22] D. Leykam, J. D. Bodyfelt, A. S. Desyatnikov, and S. Flach, Localization of weakly disordered flat band states, Eur. Phys. J. B 90, 1 (2017).

[23] C. Danieli, J. D. Bodyfelt, and S. Flach, Flat-band engineering of mobility edges, Phys. Rev. B 91, 235134 (2015).

[24] A. Radosavljević, G. Gligorić, P. P. Beličev, A. Maluckov, and M. Stepić, Light propagation in binary kagome ribbons with evolving disorder, Phys. Rev. E 96, 012225 (2017).

[25] C. Gneiting, Z. Li, and F. Nori, Lifetime of flatband states, Phys. Rev. B 98, 134203 (2018).

[26] G. Sierra, M. A. Martín-Delgado, S. R. White, D. J. Scalapino, and J. Dukelsky, Diagonal ladders: A class of models for strongly coupled electron systems, Phys. Rev. B 59, 7973 (1999).

[27] J. Vidal, B. Douçot, R. Mosseri, and P. Butaud, Interaction Induced Delocalization for Two Particles in a Periodic Potential, Phys. Rev. Lett. 85, 3906 (2000).

[28] J. Vidal, P. Butaud, B. Douçot, and R. Mosseri, Disorder and interactions in Aharonov-Bohm cages, Phys. Rev. B 64, 155306 (2001). 
[29] Z. Gulácsi, A. Kampf, and D. Vollhardt, Exact Many-Electron Ground States on the Diamond Hubbard Chain, Phys. Rev. Lett. 99, 026404 (2007).

[30] R. Mondaini, G. G. Batrouni, and B. Grémaud, Pairing and superconductivity in the flat band: Creutz lattice, Phys. Rev. B 98, 155142 (2018).

[31] Y. Kuno, T. Orito, and I. Ichinose, Flat-band many-body localization and ergodicity breaking in the creutz ladder, New J. Phys. 22, 013032 (2020).

[32] C. Danieli, A. Andreanov, T. Mithun, and S. Flach, Nonlinear caging in all-bands-flat lattices, arXiv:2004.11871 (2020).

[33] C. Danieli, A. Andreanov, T. Mithun, and S. Flach, Quantum caging in interacting many-body all-bands-flat lattices, arXiv:2004.11880 (2020).

[34] C. Danieli, A. Andreanov, and S. Flach, Many-body flatband localization, Phys. Rev. B 102, 041116(R) (2020).

[35] L. Gong and P. Tong, Fidelity, fidelity susceptibility, and von neumann entropy to characterize the phase diagram of an extended harper model, Phys. Rev. B 78, 115114 (2008).

[36] X. Jia, A. R. Subramaniam, I. A. Gruzberg, and S. Chakravarty, Entanglement entropy and multifractality at localization transitions, Phys. Rev. B 77, 014208 (2008).

[37] N. Roy and A. Sharma, Entanglement contour perspective for "strong area-law violation" in a disordered long-range hopping model, Phys. Rev. B 97, 125116 (2018).

[38] J. Eisert, M. Cramer, and M. B. Plenio, Colloquium: Area laws for the entanglement entropy, Rev. Mod. Phys. 82, 277 (2010).

[39] N. Laflorencie, Quantum entanglement in condensed matter systems, Phys. Rep. 646, 1 (2016).

[40] I. Peschel, Calculation of reduced density matrices from correlation functions, J. Phys. A: Math. Gen. 36, L205 (2003).

[41] I. Peschel and V. Eisler, Reduced density matrices and entanglement entropy in free lattice models, J. Phys. A: Math. Theor. 42, 504003 (2009).

[42] I. Peschel, Special review: Entanglement in solvable manyparticle models, Braz. J. Phys. 42, 267 (2012).
[43] N. Roy, A. Sharma, and R. Mukherjee, Quantum simulation of long-range $x y$ quantum spin glass with strong area-law violation using trapped ions, Phys. Rev. A 99, 052342 (2019).

[44] N. Roy and A. Sharma, Study of counterintuitive transport properties in the aubry-andré-harper model via entanglement entropy and persistent current, Phys. Rev. B 100, 195143 (2019).

[45] B. Swingle, Entanglement Entropy and the Fermi Surface, Phys. Rev. Lett. 105, 050502 (2010).

[46] V. Oganesyan and D. A. Huse, Localization of interacting fermions at high temperature, Phys. Rev. B 75, 155111 (2007).

[47] D. Thongjaomayum, A. Andreanov, T. Engl, and S. Flach, Taming two interacting particles with disorder, Phys. Rev. B 100, 224203 (2019).

[48] N. Macé, F. Alet, and N. Laflorencie, Multifractal Scalings Across the Many-Body Localization Transition, Phys. Rev. Lett. 123, 180601 (2019).

[49] M. Tovmasyan, S. Peotta, L. Liang, P. Törmä, and S. D. Huber, Preformed pairs in flat bloch bands, Phys. Rev. B 98, 134513 (2018).

[50] M. Di Liberto, S. Mukherjee, and N. Goldman, Nonlinear dynamics of Aharonov-Bohm cages, Phys. Rev. A 100, 043829 (2019).

[51] M. Žnidarič, T. Prosen, and P. Prelovšek, Many-body localization in the Heisenberg X X Z magnet in a random field, Phys. Rev. B 77, 064426 (2008).

[52] D. A. Abanin, E. Altman, I. Bloch, and M. Serbyn, Colloquium: Many-body localization, thermalization, and entanglement, Rev. Mod. Phys. 91, 021001 (2019).

[53] D. Wiater and J. Zakrzewski, Impact of geometry on many-body localization, Phys. Rev. B 98, 094202 (2018).

[54] E. V. H. Doggen, I. V. Gornyi, A. D. Mirlin, and D. G. Polyakov, Slow Many-Body Delocalization Beyond One Dimension, Phys. Rev. Lett. 125, 155701 (2020).

[55] S. Iyer, V. Oganesyan, G. Refael, and D. A. Huse, Many-body localization in a quasiperiodic system, Phys. Rev. B 87, 134202 (2013). 